\title{
STABILITY OF PRECISE LAPLACE'S METHOD UNDER APPROXIMATIONS; APPLICATIONS
}

\author{
Alice Guionnet ${ }^{1}$
}

\begin{abstract}
We study the fluctuations around non degenerate attractors of the empirical measure under mean field Gibbs measures. We prove that a mild change of the densities of these measures does not affect the central limit theorems. We apply this result to generalize the assumptions of [3] and [12] on the densities of the Gibbs measures to get precise Laplace estimates.

Résumé. Nous étudions les fluctuations de la mesure empirique sous une mesure de Gibbs du type champ moyen autour d'un attracteur non dégénéré. Nous montrons que les densités de ces mesures peuvent être approchées sans affecter les propriétés de fluctuations de la mesure empirique. En utilisant cette idée, nous améliorons les hypothèses de [3] et [12] permettant d'obtenir des estimations précises par la méthode de Laplace.
\end{abstract}

AMS Subject Classification. 60F10, 60F05, 60E15.

Received January 9, 1998. Revised March 3, 1999.

\section{INTRODUCTION}

The fluctuations for mean field interacting particles have been widely studied (see $[3,5,7,12,13,19,20]$...). The problem can be summarized as follows. Let $\Sigma$ be a Polish space furnished with a sigma-algebra $\mathcal{F}$, and $\mathcal{P}(\Sigma)$ be the set of probability measures on $(\Sigma, \mathcal{F})$. Let $\Gamma$ be a function on $\mathcal{P}(\Sigma)$ and $P$ be an element of $\mathcal{P}(\Sigma)$. We consider the Gibbs measure $P_{\Gamma}^{N}$ defined by

$$
P_{\Gamma}^{N}(d X):=\frac{1}{Z_{\Gamma}^{N}} \exp \left\{N \Gamma\left(\frac{1}{N} \sum_{i=1}^{N} \delta_{x_{i}}\right)\right\} d P^{\otimes N}(X)
$$

where $X=\left(x_{1}, . ., x_{N}\right) \in(\Sigma)^{N}$ and with

$$
Z_{\Gamma}^{N}=\int \exp \left\{N \Gamma\left(\frac{1}{N} \sum_{i=1}^{N} \delta_{x_{i}}\right)\right\} d P^{\otimes N}(X)
$$

Keywords and phrases: Large deviation, central limit theorem, U-statistics.

${ }^{1}$ URA 743 du CNRS, bâtiment 425, Université de Paris Sud, 91405 Orsay, France; e-mail: Alice.guionnet@math.u-psud.fr 
One is interested in the asymptotic properties of $P_{\Gamma}^{N}$ when the number $N$ of particles goes to infinity. In particular, one would like to understand the asymptotic behaviour of the empirical measure

$$
\hat{\mu}^{N}:=\frac{1}{N} \sum_{i=1}^{N} \delta_{x_{i}},
$$

that is its convergence (law of large numbers) and its fluctuations (central limit theorem). The convergence of the empirical measure is now well described. If $\Gamma$ is for instance bounded continuous as a function on $\mathcal{P}(\Sigma)$ furnished with the weak (or the strong) topology, it is well known (see [9], Ths. 4.3.1 and 6.3.1) that the law of $\hat{\mu}^{N}$ under $P_{\Gamma}^{N}$ satisfies a large deviation principle with good rate function

$$
H(\mu):=I(\mu \mid P)-\Gamma(\mu)-\inf \{I(\mid P)-\Gamma\}
$$

where $I(\mu \mid P)$ is the relative entropy of $\mu$ with respect to $P$

$$
I(\mu \mid P)=\left\{\begin{array}{lr}
\int \log \frac{d \mu}{d P} d \mu & \text { if } \mu \ll P \\
+\infty & \text { otherwise }
\end{array}\right.
$$

At least in non degenerate cases, the empirical measure converges to a convex combination of the minimizers of $H$. Let us assume for simplification that $H$ achieves its minimal value at a unique probability measure $\mu^{*}$ which is a non degenerate minimum (that is, roughly speaking, that $H$ is strictly convex in a neighborhood of $\mu^{*}$ (see Th. 1.1 for a precise statement)). Then, one expects the fluctuations around $\mu^{*}$ to be Gaussian. This type of result was indeed proven for various functions $\Gamma$ in $[3,5,7,12,13,19,20]$. To our knowledge, the most general assumptions on $\Gamma$ can be found in [3] or [12] (except for dynamical densities where $Z_{\Gamma}^{N} \equiv 1$ which is treated in $[17])$. In both articles, the authors assume that $\Gamma$ is of the form

$$
\Gamma(\mu):=\sum_{k=2}^{r} \int V_{k}\left(x_{1}, . ., x_{k}\right) d \mu^{\otimes k}\left(x_{1}, . ., x_{k}\right)
$$

for a finite integer number $r$ and functions $V_{k}$ on $\Sigma^{k}$ so that there exists a compact measured space $(C, \nu)$ and a bounded continuous function $g: C \times \Sigma \rightarrow \mathbb{R}$ so that, for any $k \in\{1, . ., r\}$ and any $\left(x_{1}, . ., x_{k}\right) \in \Sigma^{k}$,

$$
V_{k}\left(x_{1}, . ., x_{k}\right)=\int g\left(\tau, x_{1}\right) g\left(\tau, x_{2}\right) . . g\left(\tau, x_{k}\right) d \nu(\tau) .
$$

This assumption is crucial in [3] to map continuously $\mathcal{P}(\Sigma)$ furnished with the weak topology into the Banach space $\mathcal{B}(\Gamma)=L^{r}(\nu)$ by $T_{V}(\mu)(\tau)=\int g(\tau, x) d \mu(x)$ in order to use the work of Bolthausen [5].

To state the result proved in [3] and [12], let us define the Hessian $\Xi$ of $\Gamma$, that is the symmetric operator in the subspace $L_{0}^{2}\left(\mu^{*}\right)=\left\{\phi \in L^{2}\left(\mu^{*}\right) ; \int \phi d \mu^{*}=0\right\}$ of $L^{2}\left(\mu^{*}\right)$ so that, for any $\phi \in L_{0}^{2}\left(\mu^{*}\right)$ such that for $\epsilon \in \mathbb{R}$ small enough $(1+\epsilon \phi) \cdot \mu^{*} \in \mathcal{P}(\Sigma)$,

$$
\langle\phi, \Xi \phi\rangle_{L_{\mu^{*}}^{2}}=\lim _{\epsilon \downarrow 0} \frac{1}{2 \epsilon^{2}}\left\{\Gamma\left((1+\epsilon \phi) \cdot \mu^{*}\right)+\Gamma\left((1-\epsilon \phi) \cdot \mu^{*}\right)-2 \Gamma\left(\mu^{*}\right)\right\} .
$$

The authors then proved in [3] and [12] that if $\Gamma$ satifies (1),

Theorem 1.1. If $\mu^{*}$ is not degenerate, that is if $I-\Xi$ is positive definite, for any $f \in L_{0}^{2}\left(\mu^{*}\right),(1 / \sqrt{N}) \sum_{i=1}^{N} f\left(x_{i}\right)$ converges in law under $P_{\Gamma}^{N}$ to a centered Gaussian variable with covariance

$$
\sigma(f)=\left\langle f,(I-\Xi)^{-1} f\right\rangle_{L^{2}\left(\mu^{*}\right)} .
$$


Our purpose here is to propose a method to relax the assumptions on the functions $\Gamma$ for non degenerate minimizer $\mu^{*}$. To simplify, we will assume as well that $\mu^{*}$ is the unique minimizer of $H$. However, the reader can easily extend our results to the case where $H$ has several non degenerate minimizers but when he considers the probability $P_{\Gamma}^{N}$ conditioned by the event that the empirical measure remains in a small neighborhood of one minimizer (such local central limit theorems were studied in [3] and in [12] (see Lem. 3.2)). Indeed, all the proofs of this type of results use first a localization around the minimizer which boils down to consider the probability $P_{\Gamma}^{N}$ conditioned by the event that the empirical measure stays in a small neighborhood of $\mu^{*}$. We will assume in the following:

$(\mathrm{H} 0) P_{\Gamma}^{N}$ satisfies a large deviation principle with good rate function $H$ for the strong topology. $H$ achieves its minimum value at a unique probability measure $\mu^{*} . \mu^{*}$ is a non degenerate minimum of $H$.

We will extend the study of the fluctuations for functions $\Gamma$ which are of the form (1) but with more general functions $V_{k}$ 's (in particular, functions which do not satisfy (2)). Then, we will tackle the case of "analytic" functions $\Gamma$, that is of functions $\Gamma$ of type (1) but with $r=\infty$.

To be more precise, let us introduce a few extra definitions. Let $k$ be an integer number. We will say in the sequel that a function $F$ on $\Sigma^{k}$ is bounded $\mu^{*}$-canonical if it is a bounded measurable function such that $\int F\left(x_{1}, . ., x_{k}\right) d \mu^{*}\left(x_{i}\right)=0$ for any $i \in\{1, . ., k\}$ and any $\left(x_{j}\right)_{j \neq i} \in \Sigma^{k-1}$. Moreover, we will say that a bounded function $F$ is regular if $F$ is a bounded measurable function such that the maps $\psi_{F}^{1}$ and $\psi_{F}^{2}: \mathcal{P}(\Sigma \times\{-1,+1\})$ $\rightarrow \mathbb{R}$ defined by

$$
\psi_{F}^{1}(\mu)=\int F(x, y) \epsilon \widetilde{\epsilon} d \mu(x, \epsilon) d \mu(y, \widetilde{\epsilon})
$$

and

$$
\psi_{F}^{2}(\mu)=\int F(x, y)^{2} d \mu(x, \epsilon) d \mu(y, \widetilde{\epsilon})
$$

are bounded continuous for the strong topology. Note that if $F$ is continuous, $F$ is regular according to Lemma 7.3.12 of [9]. However, $F$ is also regular if it is the limit for the uniform topology of functions of type $\sum_{i=1}^{s} \alpha_{i} \phi_{i}(x) \psi_{i}(y)$ for a finite integer number $s$ and bounded measurable functions $\phi_{i}$ 's and $\psi_{i}$ 's. However, a bounded measurable function $F$ can fell to be regular (see Ex. 7.3.18 in [9]).

Let us state the main result of this paper

Theorem 1.2. Assume $\Gamma$ of the form

$$
\Gamma(\mu)=\sum_{k=0}^{\infty} \frac{1}{k !} \int W_{k}\left(x_{1}, . ., x_{k}\right) d(\mu)^{\otimes k}\left(x_{1}, . ., x_{k}\right)
$$

with symmetric bounded $\mu^{*}$-canonical functions $W_{k}$ so that

1) the $W_{k}$ 's satisfy, for some universal constant $c>0$ small enough and any $k \geq 0$,

$$
\left\|W_{k}\right\|_{\infty} \leq \text { const. } \sqrt{k !} c^{k}
$$

2) $W_{2}$ is bounded regular,

then, if (HO) is fulfilled, the conclusions of Theorem 1.1 are valid.

As one can see, our generalization is based on some regularity properties of the function $\Gamma$ rather than on some structural hypothesis of type (2). In particular, $\Gamma$ has to have all its Frechet derivatives (see Sect. 2 for a definition).

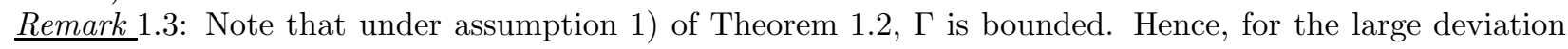
principle to hold with good rate function $H$, it is enough to add the hypothesis that $\Gamma$ is continuous for the strong topology (see [9], Ths. 4.3.1 and 6.3.1). 
In any case, our approach is build upon the control of sufficiently good approximations of the functions $\Gamma$. Namely, if $\left(\Gamma_{\epsilon}\right)_{\epsilon>0}$ is a sequence of functions on $\Sigma$ converging to $\Gamma$ sufficiently well for which we know that Theorem 1.1 holds, we will prove that for any bounded continuous functions $F: \mathbb{R} \rightarrow \mathbb{R}$ and any measurable function $f: \Sigma \rightarrow \mathbb{R}$ in $L_{0}^{2}\left(\mu^{*}\right)$,

$$
\lim _{N \rightarrow \infty} \int F\left(\frac{1}{\sqrt{N}} \sum_{i=1}^{N} f\left(x_{i}\right)\right) d P_{\Gamma}^{N}=\lim _{\epsilon \downarrow 0} \lim _{N \rightarrow \infty} \int F\left(\frac{1}{\sqrt{N}} \sum_{i=1}^{N} f\left(x_{i}\right)\right) d P_{\Gamma_{\epsilon}}^{N} .
$$

Again, if the $\left(\Gamma_{\epsilon}\right)_{\epsilon>0}$ satisfy (2) and approximate sufficiently well $\Gamma$ as $\epsilon \downarrow 0$, one can compute the r.h.s. of (3) and conclude. (3) is therefore the main point in this paper. It is not a priori clear since the error one should make by a trivial bound should be of order $e^{o(N)}$. Its proof is driven by the control on $U$-statistics developed by De La Peña [15] and Arcones-Gine [1].

The article is organized as follows.

In Section 2, we develop the approximation scheme for polynomial functions $\Gamma$.

In Section 3, we prove the crucial estimates to control our approximations.

In Section 4, we apply this strategy for analytic functions $\Gamma$ 's.

\section{Polynomial interaction}

Throughout this section, we will consider real valued functions $\Gamma$ on $\mathcal{P}(\Sigma)$ so that there exists bounded measurable functions $\left(V_{k}\right)_{k \geq 0}$ so that

$$
\Gamma(\mu)=\sum_{k=0}^{r} \frac{1}{k !} \int V_{k} d(\mu)^{\otimes k}
$$

We shall often use the $\mu^{*}$-canonical decomposition of $\Gamma$, that is write $\Gamma$ in terms of bounded $\mu^{*}$-canonical symmetric functions $\left(W_{k}\right)_{k \geq 0}$ as

$$
\Gamma(\mu)=\sum_{k=0}^{r} \frac{1}{k !} \int W_{k} d(\mu)^{\otimes k}
$$

The main result of this section then states as follows

Theorem 2.1. Assume that $W_{2}$ is bounded regular and that the $W_{k}$ 's are bounded. Then, for any bounded continuous function $f$ so that $\int f d \mu^{*}=0,(1 / \sqrt{N}) \sum_{i=1}^{N} f\left(x_{i}\right)$ converges under $P_{\Gamma}^{N}$ to a centered Gaussian variable with covariance

$$
\sigma(f)=\left\langle f,(I-\Xi)^{-1} f\right\rangle_{L^{2}\left(\mu^{*}\right)}
$$

To prove Theorem 2.1, let us first state precisely our approximation result caricatured by (3). To this end, let us introduce the following approximation property

(H1) There exists a sequence $W_{\epsilon}$ of continuous symmetric functions on $\Sigma \times \Sigma$ so that $\int W_{\epsilon}\left(x_{1}, x_{2}\right) d \mu^{*}\left(x_{1}\right) \equiv 0$ for any $x_{2} \in \Sigma$ and satisfying

$$
\begin{aligned}
\lim _{\epsilon \downarrow 0} \int\left(W_{2}-W_{\epsilon}\right)^{2}(x, y) d \mu^{*}(x) d \mu^{*}(y) & =0, \\
\lim _{\epsilon \downarrow 0} \int\left(W_{2}-W_{\epsilon}\right)(x, x) d \mu^{*}(x) & =0 .
\end{aligned}
$$

Moreover, $W_{\epsilon}$ is uniformly bounded, that is, if \|\|$_{\infty}$ denotes the uniform norm,

$$
\sup _{\epsilon}\left\|W_{\epsilon}\right\|_{\infty}<\infty
$$

and, for any $\epsilon>0, W_{\epsilon}$ satisfies the hypothesis of [3] or [12] (see its statement at (2)). 
We shall prove in the appendix, Lemma 5.4, that any bounded measurable function $W_{2}$ satisfies (H1). Hereafter, we shall fix a family of functions $\left(W_{\epsilon}\right)_{\epsilon>0}$ satisfying $(\mathrm{H} 1)$ and note $\Gamma_{\epsilon}: \mathcal{P}(\Sigma) \rightarrow \mathbb{R}$

$$
\Gamma_{\epsilon}(\mu)=\frac{1}{2} \int W_{\epsilon}(x, y) d \mu(x) d \mu(y) .
$$

We shall see in this section that

Theorem 2.2. Under (HO) and (H1), if $W_{2}$ is bounded regular and the $W_{k}$ 's bounded, for any bounded continuous functions $F$ and any $f \in L_{0}^{2}\left(\mu^{*}\right)$,

$$
\lim _{N \rightarrow \infty} \int F\left(\frac{1}{\sqrt{N}} \sum_{i=1}^{N} f\left(x_{i}\right)\right) d P_{\Gamma}^{N}=\lim _{\epsilon \downarrow 0} \lim _{N \uparrow \infty} \int F\left(\frac{1}{\sqrt{N}} \sum_{i=1}^{N} f\left(x_{i}\right)\right) d P_{\Gamma_{\epsilon}}^{N} .
$$

Note

$$
\widetilde{\Gamma}(\mu):=\Gamma(\mu)-\Gamma\left(\mu^{*}\right)-D \Gamma\left[\mu^{*}\right]\left(\mu-\mu^{*}\right)=\sum_{k=2}^{r} \frac{1}{k !} \int W_{k} d \mu^{\otimes k}
$$

Since $\mu^{*}$ minimizes $I(. \mid P)-\Gamma$, it is not hard to check (see [3], for details) that

$$
\log \frac{d \mu^{*}}{d P}=D \Gamma\left[\mu^{*}\right]\left(\delta_{x}\right)+\text { const. }
$$

where $D$ is the Frechet derivative

$$
D \Gamma\left[\mu^{*}\right]\left(\nu-\mu^{*}\right):=\lim _{\delta \rightarrow 0} \frac{1}{\delta}\left(\Gamma\left((1-\delta) \mu^{*}+\delta \nu\right)-\Gamma\left(\mu^{*}\right)\right) .
$$

Hence,

$$
d P_{\Gamma}^{N}(X)=\frac{1}{\widetilde{Z}_{N}} \exp \left\{N \widetilde{\Gamma}\left(\hat{\mu}^{N}\right)\right\} d\left(\mu^{*}\right)^{\otimes N}(X)
$$

with

$$
\widetilde{Z}_{N}=\int \exp \left\{N \widetilde{\Gamma}\left(\hat{\mu}^{N}\right)\right\} d\left(\mu^{*}\right)^{\otimes N}(X) .
$$

To prove Theorem 2.2, we shall first consider the partition function $\widetilde{Z}_{N}$. When $\Gamma$ satisfies (1), it is well known (see [12], Th. 1, for instance) that

$$
\lim _{N \rightarrow \infty} \widetilde{Z}_{N}=(\operatorname{det}(I-\Xi))^{-\frac{1}{2}} .
$$

Because our assumption on $W_{2}$ does not insure that $\Xi$ is trace class, and therefore that the above determinant is well defined, we are not going to prove such a result here. However, if we assume also that $\Xi$ is trace class, then, according to [4], $\operatorname{tr}(\Xi)=\int W(x, x) d \mu(x)$ and (H1) insures that $\lim _{\epsilon \downarrow 0} \operatorname{tr}\left(\Xi_{\epsilon}\right)=\operatorname{tr}(\Xi)$. Thus, we could use the lemmas below to show that (8) holds. Instead, we are going to provide bounds on $\widetilde{Z}^{N}$ in terms of determinants of the operators $\Xi_{\epsilon}$ in $L_{0}^{2}\left(\mu^{*}\right)$ with kernel $W_{\epsilon}$;

$$
\left\langle\Xi_{\epsilon} \phi, \psi\right\rangle_{L^{2}\left(\mu^{*}\right)}=\int W_{\epsilon}\left(x_{1}, x_{2}\right) \phi\left(x_{1}\right) \psi\left(x_{2}\right) d \mu^{*}\left(x_{1}\right) d \mu^{*}\left(x_{2}\right)
$$


for $(\phi, \psi) \in L_{0}^{2}\left(\mu^{*}\right)$. $\Xi_{\epsilon}$ is trace class according to the hypothesis on $W_{\epsilon}$ (see [3]) for every $\epsilon>0$. To obtain Theorems 2.1 and 2.2, we shall use some cancellation to get bounds depending only on the regularized determinant $\operatorname{det}_{2}$ defined, for any operator $A$ on $L^{2}\left(\mu^{*}\right)$, by

$$
\operatorname{det}_{2}(I-A)=e^{-\operatorname{tr}(A)} \operatorname{det}(I-A)
$$

which is continuous for the Hilbert-Schmidt norm defined, for any operator $A$ in $L^{2}\left(\mu^{*}\right)$, by

$$
\|A\|_{H S}=\sqrt{\operatorname{tr}\left(A^{*} A\right)} .
$$

We will then pass to the limit thanks to (H1). In fact, note that if $\Xi$ is the operator in $L_{0}^{2}\left(\mu^{*}\right)$ with kernel $W_{2}$ so that

$$
\left\langle\Xi_{\epsilon} \phi, \psi\right\rangle_{L^{2}\left(\mu^{*}\right)}=\int W_{\epsilon}\left(x_{1}, x_{2}\right) \phi\left(x_{1}\right) \psi\left(x_{2}\right) d \mu^{*}\left(x_{1}\right) d \mu^{*}\left(x_{2}\right)
$$

for $(\phi, \psi) \in L_{0}^{2}\left(\mu^{*}\right) ;(4)$ reads

$$
\lim _{\epsilon \downarrow 0}\left\|\Xi-\Xi_{\epsilon}\right\|_{H S}^{2}=\lim _{\epsilon \downarrow 0} \operatorname{tr}\left(\left(\Xi-\Xi_{\epsilon}\right)\left(\Xi-\Xi_{\epsilon}\right)^{*}\right)=0 .
$$

Consequently, since $I-\Xi$ is positive definite, $I-\Xi_{\epsilon}$ is also positive definite for $\epsilon$ small enough. Also, $\operatorname{det}_{2}\left(I-\Xi_{\epsilon}\right)$ converges towards $\operatorname{det}_{2}\left(I-\Xi_{\epsilon}\right)$ as $\epsilon$ goes to zero. For the same reasons, there exists $\alpha_{0}>1$ so that for $\alpha<\alpha_{0}$ and $\epsilon$ small enough, $\epsilon<\epsilon(\alpha), I-\alpha \Xi_{\epsilon}$ is still positive definite. In particular, $\operatorname{det}_{2}\left(I-\alpha \Xi_{\epsilon}\right)$ converges, as $\alpha \downarrow 1$ and $\epsilon \downarrow 0$ towards $\operatorname{det}_{2}(I-\Xi)$. In the following, we will fix $\alpha \in\left(1, \alpha_{0}\right)$ and assume $\epsilon<\epsilon(\alpha)$.

In view of the above considerations, the following bounds will be meaningful and useful

Lemma 2.3. a) If $\alpha>1$ is small enough, there exists $\epsilon(\alpha)>0$ so that, for any $\epsilon<\epsilon(\alpha)$,

$$
\limsup _{N \rightarrow \infty} \widetilde{Z}_{N} \leq\left(\operatorname{det}\left(I-\alpha \Xi_{\epsilon}\right)\right)^{-\frac{1}{2 \alpha}} \exp \left\{\frac{1}{2} \int\left(W_{2}-W_{\epsilon}\right)(x, x) d \mu^{*}(x)\right\} .
$$

b) For any $\eta>0$, any $A>0$, and for $\alpha>1$ small enough, any $\epsilon>0$ small enough (depending on $A$ and $\alpha$ )

$$
\liminf _{N \rightarrow \infty} \widetilde{Z}_{N} \geq e^{-\eta} \operatorname{det}\left(I-\Xi_{\epsilon}\right)^{-\frac{1}{2}}-e^{-\eta A} e^{(A / 2) \int\left(W_{2}-W_{\epsilon}\right)(x, x) d \mu^{*}(x)} \operatorname{det}\left(I-\alpha \Xi_{\epsilon}\right)^{-\frac{1}{2 \alpha}} .
$$

More generally, if $F$ is a non negative continuous function and $f \in L_{0}^{2}\left(\mu^{*}\right)$, define

we shall see that if

$$
\widetilde{Z}_{N}^{F, f}=\iint F\left(\frac{1}{\sqrt{N}} \sum_{i=1}^{N} f\left(x_{i}\right)\right) e^{N \widetilde{\Gamma}\left(\hat{\mu}^{N}\right)} d\left(\mu^{*}\right)^{\otimes N}(X),
$$

$$
\sigma_{\epsilon}(f):=\left\langle f,\left(I-\Xi_{\epsilon}\right)^{-1} f\right\rangle_{L^{2}\left(\mu^{*}\right)} .
$$

Lemma 2.4. Let $F$ be a non negative continuous function and $f \in L_{0}^{2}\left(\mu^{*}\right)$, a) if $\alpha>1$ is small enough, there exists $\epsilon(\alpha)>0$ so that, for any $\epsilon<\epsilon(\alpha)$,

$$
\limsup _{N \rightarrow \infty} \widetilde{Z}_{N}^{F, f} \leq e^{\frac{1}{2} \int\left(W_{2}-W_{\epsilon}\right)(x, x) d \mu^{*}(x)}\left(\frac{1}{\sqrt{2 \pi \operatorname{det}\left(I-\alpha \Xi_{\epsilon}\right) \sigma_{\epsilon}(f)}} \int F^{\alpha}(x) e^{-\frac{x^{2}}{2 \sigma_{\epsilon}(f)}} d x\right)^{\frac{1}{\alpha}} .
$$

b) For any $\eta>0$, any $A>0$, and for $\alpha>1$ small enough, any $\epsilon>0$ small enough (depending on $A$ and $\alpha$ )

$$
\begin{aligned}
\liminf _{N \rightarrow \infty} \widetilde{Z}_{N}^{F, f} & \geq e^{-\eta} \frac{1}{\sqrt{2 \pi \operatorname{det}\left(I-\Xi_{\epsilon}\right) \sigma_{\epsilon}(f)}} \int F(x) e^{-\frac{x^{2}}{2 \sigma_{\epsilon}(f)}} d x \\
& -e^{-\eta A} e^{(A / 2) \int\left(W_{2}-W_{\varepsilon}\right)(x, x) d \mu^{*}(x)}\|F\|_{\infty} \operatorname{det}\left(I-\alpha \Xi_{\epsilon}\right)^{-\frac{1}{2 \alpha}} .
\end{aligned}
$$


Let us first derive Theorems 2.1 and 2.2 from Lemmas 2.3 and 2.4. In fact, we need to prove that

$$
\begin{aligned}
\Lambda_{N}^{F, f} & :=\lim _{N \rightarrow \infty} \int F\left(\frac{1}{\sqrt{N}} \sum_{i=1}^{N} f\left(x_{i}\right)\right) e^{N \widetilde{\Gamma}\left(\hat{\mu}^{N}\right)} d\left(\mu^{*}\right)^{\otimes N}(X)=\lim _{N \rightarrow \infty} \frac{\widetilde{Z}_{N}^{F, f}}{\widetilde{Z}_{N}} \\
& =\frac{1}{\sqrt{2 \pi \sigma(f)}} \int F(x) e^{-\frac{x^{2}}{2 \sigma(f)}} d x
\end{aligned}
$$

for any bounded continuous function $F$. Without loss of generality, we can assume $F$ non negative.

By Lemma 2.3b) and Lemma 2.4a), we first get that for any $\eta>0$, any $A>0$, and for $\alpha>1$ small enough, any $\epsilon>0$ small enough (so that the r.h.s. of Lem. 2.3b) is positive),

$$
\limsup _{N \rightarrow \infty} \Lambda_{N}^{F, f} \leq \frac{\exp \left\{\frac{1}{2} \int\left(W_{2}-W_{\epsilon}\right)(x, x) d \mu^{*}(x)\right\}}{e^{-\eta}\left(\frac{\operatorname{det}\left(I-\alpha \Xi_{\epsilon}\right)^{\frac{1}{\alpha}}}{\operatorname{det}\left(I-\Xi_{\epsilon}\right)}\right)^{\frac{1}{2}}-e^{-\eta A+0.5 A \int\left(W_{2}-W_{\epsilon}\right)(x, x) d \mu^{*}(x)}} \times\left(\frac{1}{\sqrt{2 \pi \sigma_{\epsilon}(f)}} \int F^{\alpha}(x) e^{-\frac{x^{2}}{2 \sigma_{\epsilon}(f)}} d x\right)^{\frac{1}{\alpha}} .
$$

(H1) results with

$$
\lim _{\epsilon \downarrow 0} \int\left(W_{2}-W_{\epsilon}\right)(x, x) d \mu^{*}(x)=0 .
$$

Further,

$$
\frac{\operatorname{det}\left(I-\alpha \Xi_{\epsilon}\right)^{\frac{1}{\alpha}}}{\operatorname{det}\left(I-\Xi_{\epsilon}\right)}=\frac{\operatorname{det}_{2}\left(I-\alpha \Xi_{\epsilon}\right)^{\frac{1}{\alpha}}}{\operatorname{det}_{2}\left(I-\Xi_{\epsilon}\right)} .
$$

It is well known (see [18], Th. 9.2c)) that the regularized determinant $\operatorname{det}_{2}$ is continuous for the Hilbert-Schmidt norm \|\|$_{H S}$. Therefore, since assumption (4) shows that $\Xi_{\epsilon}$ converges in the Hilbert-Schmidt topology to $\Xi$, we deduce that

$$
\lim _{\alpha \downarrow 1} \lim _{\epsilon \downarrow 0} \frac{\operatorname{det}_{2}\left(I-\alpha \Xi_{\epsilon}\right)^{\frac{1}{\alpha}}}{\operatorname{det}_{2}\left(I-\Xi_{\epsilon}\right)}=\lim _{\alpha \downarrow 1} \frac{\operatorname{det}_{2}(I-\alpha \Xi)^{\frac{1}{\alpha}}}{\operatorname{det}_{2}(I-\Xi)}=1 .
$$

Moreover, by construction, we can find a positive constant $a$ so that $\left(I-\Xi_{\epsilon}\right) \geq a I$ for $\epsilon$ small enough. Hence the convergence of $\Xi_{\epsilon}$ towards $\Xi$ for the HS-topology results with

$$
\lim _{\epsilon \rightarrow 0} \sigma_{\epsilon}(f)=\sigma(f)
$$

Equations (16-18) and (19) imply, once $\epsilon \downarrow 0$, that for any $\eta>0$, any $A>0$, and for $\alpha>1$ small enough,

$$
\limsup _{N \rightarrow \infty} \Lambda_{N}^{F, f} \leq \frac{1}{e^{-\eta}\left(\frac{\operatorname{det}\left(I-\alpha \Xi \frac{1}{\alpha}\right.}{\operatorname{det}(I-\Xi)}\right)^{\frac{1}{2}}-e^{-\eta A}}\left(\frac{1}{\sqrt{2 \pi \sigma(f)}} \int F(x) e^{-\frac{x^{2}}{2 \sigma(f)}} d x\right)^{\frac{1}{\alpha}}
$$

Letting $\alpha \downarrow 1, A \uparrow \infty$ and $\eta \downarrow 0$ give

$$
\limsup _{N \rightarrow \infty} \widetilde{Z}_{N}^{F, f} \leq \frac{1}{\sqrt{2 \pi \sigma(f)}} \int F(x) e^{-\frac{x^{2}}{2 \sigma(f)}} d x .
$$


We proceed similarly for the lower bound; in view of Lemmas $2.3 \mathrm{a}$ ) and $2.4 \mathrm{~b}$ ), we find that for any $\eta>0$, any $A>0$, and for $\alpha>1$ small enough (so that the r.h.s. of Lem. 2.4b) is positive), any $\epsilon>0$ small enough,

$$
\begin{gathered}
\liminf _{N \rightarrow \infty} \Lambda_{N}^{F, f} \geq e^{-\eta-\frac{1}{2} \int\left(W_{2}-W_{\epsilon}\right)(x, x) d \mu^{*}(x)}\left(\frac{\operatorname{det}\left(I-\alpha \Xi_{\epsilon}\right)^{\frac{1}{\alpha}}}{2 \pi \operatorname{det}\left(I-\Xi_{\epsilon}\right) \sigma_{\epsilon}(f)}\right)^{\frac{1}{2}} \int F(x) e^{-\frac{x^{2}}{2 \sigma \epsilon(f)}} d x \\
-e^{-\eta A+\frac{A}{2} \int\left(W_{2}-W_{\epsilon}\right)(x, x) d \mu^{*}(x)}\|F\|_{\infty} .
\end{gathered}
$$

Consequently $(17,18)$ and (19) imply, once $\epsilon \downarrow 0, \alpha \downarrow 1, A \uparrow \infty$ and $\eta \downarrow 0$,

$$
\liminf _{N \rightarrow \infty} \Lambda_{N}^{F, f} \geq \frac{1}{\sqrt{2 \pi \sigma(f)}} \int F(x) e^{-\frac{x^{2}}{2 \sigma(f)}} d x
$$

which completes the proof of Theorems 2.1 and 2.2 .

Hence, we need to prove Lemma 2.4. An essential and basic consideration should be made before beginning this proof. Under our large deviation hypothesis, the probability that the empirical measure does not belong to an open set containing $\mu^{*}$ is exponentially small. Among the open neighborhood of $\mu^{*}$, we shall be specially interested in those inherited from the Banach spaces $\mathcal{B}\left(\Gamma_{\epsilon}\right)$ attached to the functions $W_{\epsilon}$ (see assumption (2)). Namely, if $\Gamma_{\epsilon}(\mu)=\int\left(\int g_{\epsilon}(x, \tau) d \mu(x)\right)^{2} d \nu_{\epsilon}(\tau)$ with bounded continuous $\mu^{*}$-canonical functions $\left(g_{\epsilon}(., \tau)\right.$, $\epsilon>0, \tau \in C), \mathcal{B}\left(\Gamma_{\epsilon}\right)=L^{2}\left(\nu_{\epsilon}\right)$ and $T_{\epsilon}: \mathcal{P}(\Sigma) \rightarrow \mathcal{B}\left(\Gamma_{\epsilon}\right)$ is defined by $T_{\epsilon}(\mu)=\int g_{\epsilon}(x,). d \mu(x)$, we set

$$
B_{\delta}^{\epsilon}\left(\mu^{*}\right)=\left\{\mu \in \mathcal{P}(\Sigma):\left\|T_{\epsilon} \mu\right\|_{\epsilon}<\delta\right\}
$$

with \|\|$_{\epsilon}=\|\|_{L^{2}\left(\nu_{\epsilon}\right)}$. $B_{\delta}^{\epsilon}\left(\mu^{*}\right)$ is an open neighborhood of $\mu^{*}$ for the strong topology. If $\mathbb{E}$ denotes the expectation under $\left(\mu^{*}\right)^{\otimes N}$, our large deviation hypothesis implies, since $B_{\delta}^{\epsilon}\left(\mu^{*}\right)^{c}$ is a closed subset of $\mathcal{P}(\Sigma)$, that for any $\delta>0$,

$$
\limsup _{N \rightarrow \infty} \frac{1}{N} \log \mathbb{E}\left[\mathbb{I}_{B_{\delta}^{\epsilon}\left(\mu^{*}\right)^{c}} e^{N \widetilde{\Gamma}\left(\hat{\mu}^{N}\right)}\right] \leq-\inf _{B_{\delta}^{\epsilon}\left(\mu^{*}\right)^{c}} H .
$$

Since $H$ is a good rate function, it achieves its minimum value on $B_{\delta}^{\epsilon}\left(\mu^{*}\right)^{c}$. Thus, $\inf _{B_{\delta}^{\epsilon}\left(\mu^{*}\right)^{c}} H$ is strictly positive since $B_{\delta}^{\epsilon}\left(\mu^{*}\right)^{c}$ does not contain $\mu^{*}$. Hence, for any $\delta>0$ and $\epsilon>0$, there exists a positive constant $C$ so that for $N$ large enough, for any bounded measurable function $F$,

$$
\left|\mathbb{E}\left[F \exp \left\{N \widetilde{\Gamma}\left(\hat{\mu}^{N}\right)\right\}\right]-\mathbb{E}\left[\mathbb{I}_{B_{\delta}^{\epsilon}\left(\mu^{*}\right)} F \exp \left\{N \widetilde{\Gamma}\left(\hat{\mu}^{N}\right)\right\}\right]\right| \leq e^{-C N}\|F\|_{\infty} .
$$

In the following, we will therefore concentrate, for a fixed bounded continuous non negative function $F$ and a measurable $f \in L_{0}^{2}\left(\mu^{*}\right)$, on

$$
\widetilde{Z}_{N}^{\delta, \epsilon}:=\mathbb{E}\left[\mathbb{I}_{B_{\delta}^{\epsilon}\left(\mu^{*}\right)} F\left(\frac{1}{\sqrt{N}} \sum_{i=1}^{N} f\left(x_{i}\right)\right) \exp \left\{N \widetilde{\Gamma}\left(\hat{\mu}^{N}\right)\right\}\right] .
$$

Let us turn to the proof of the lemma

Proof of Lemma 2.4a). According to the previous considerations, it is enough to show that if $\alpha>1$ is small enough, there exist $\epsilon(\alpha)>0$ and $\delta(\alpha)>0$ so that, for any $\epsilon<\epsilon(\alpha)$ and $\delta<\delta(\alpha)$,

$$
\limsup _{N \rightarrow \infty} \widetilde{Z}_{N}^{\delta, \epsilon} \leq \exp \left\{\frac{1}{2} \int\left(W_{2}-W_{\epsilon}\right)(x, x) d \mu^{*}(x)\right\}\left(\frac{1}{\sqrt{2 \pi \operatorname{det}\left(I-\alpha \Xi_{\epsilon}\right) \sigma_{\epsilon}(f)}} \int F^{\alpha}(x) e^{-\frac{x^{2}}{2 \sigma_{\epsilon}(f)}} d x\right)^{\frac{1}{\alpha}} .
$$


To prove (25), note that if $\gamma$ is the conjugate exponent of $\alpha$, we have, by Hölder's inequality,

$$
\widetilde{Z}_{N}^{\delta, \epsilon} \leq L_{N}(\delta, \alpha, \epsilon)^{\frac{1}{\alpha}} R_{N}(\gamma, \epsilon)^{\frac{1}{\gamma}}
$$

with

$$
\begin{aligned}
L_{N}(\delta, \alpha, \epsilon) & :=\mathbb{E}\left[\mathbb{I}_{B_{\delta}^{\epsilon}\left(\mu^{*}\right)} F\left(\frac{1}{\sqrt{N}} \sum_{i=1}^{N} f\left(x_{i}\right)\right)^{\alpha} \exp \left\{N \frac{\alpha}{2} \int W_{\epsilon} d\left(\hat{\mu}^{N}-\mu^{*}\right)^{\otimes 2}\right\}\right] \\
R_{N}(\gamma, \epsilon) & :=\mathbb{E}\left[\exp \left\{N \frac{\gamma}{2} \int\left(W_{2}-W_{\epsilon}\right) d\left(\hat{\mu}^{N}\right)^{\otimes 2}+N \gamma \sum_{k=3}^{r} \frac{1}{k !} \int W_{k} d\left(\hat{\mu}^{N}\right)^{\otimes k}\right\}\right] .
\end{aligned}
$$

Let us first bound $L_{N}(\delta, \alpha, \epsilon)$

Lemma 2.5. For $\delta$ and $\epsilon<\epsilon(\alpha)$ small enough,

$$
\lim _{N \rightarrow \infty} L_{N}(\delta, \alpha, \epsilon)=\frac{1}{\sqrt{2 \pi \operatorname{det}\left(I-\alpha \Xi_{\epsilon}\right) \sigma_{\epsilon}(f)}} \int F^{\alpha}(x) e^{-\frac{x^{2}}{2 \sigma_{\epsilon}(f)}} d x .
$$

Proof. Following [12], Lemma 3.2, Lemma 2.5 is verified as soon as we can prove that

$$
H_{\epsilon}(\mu):=I\left(\mu \mid \mu^{*}\right)-\frac{\alpha}{2} \int W_{\epsilon} d\left(\mu-\mu^{*}\right)^{\otimes 2}
$$

achieves its minimum value at $\mu^{*}$ in $B_{\delta}^{\epsilon}\left(\mu^{*}\right)$ (so that its minimum value is in fact zero), that $\mu^{*}$ is in fact its only minimizer in $B_{\delta}^{\epsilon}\left(\mu^{*}\right)$ and that it is non degenerate. This last point is already insured by hypothesis (H1) as quoted before Lemma 2.4. For the first point, notice that, following our hypotheses, $H_{\epsilon}$ is a good rate function. It achieves its minimum value and has null derivative at $\mu^{*}$. Also, $H_{\epsilon}$ is strictly convex in a neighborhood of $\mu^{*}$ (since $I-\alpha \Xi_{\epsilon}$ is positive definite). Thus, if $\delta$ is small enough (depending eventually on $\epsilon$ and $\alpha>1$ ), $H_{\epsilon}$ achieves its minimum value uniquely at $\mu^{*}$.

Let us now consider $R_{N}(\gamma, \epsilon)$ and prove

Lemma 2.6. For any real number $\gamma$, there exists a positive $\epsilon(\gamma)$ so that, for any $\epsilon<\epsilon(\gamma)$,

$$
\limsup _{N \rightarrow \infty} R_{N}(\gamma, \epsilon) \leq \exp \left\{\frac{\gamma}{2} \int\left(W_{2}-W_{\epsilon}\right)(x, x) d \mu^{*}(x)\right\}
$$

Proof. Denote

$$
U_{N}^{k}(X)=\frac{1}{N^{k}} \sum_{i_{1}<i_{2} \ldots<i_{k}} W_{k}\left(x_{i_{1}}, . ., x_{i_{k}}\right) \quad \text { for } \quad k \geq 3, \quad U_{N}^{2}(X)=\frac{1}{N^{2}} \sum_{i_{1}<i_{2}}\left(W_{2}-W_{\epsilon}\right)\left(x_{i_{1}}, x_{i_{2}}\right),
$$

and

$$
Z_{N}(X)=\frac{1}{2 N^{2}} \sum_{i=1}^{N}\left(W_{2}-W_{\epsilon}\right)\left(x_{i}, x_{i}\right)+\sum_{k=3}^{r} \frac{1}{2(k-2) ! N^{k}} \sum_{i_{1}, . ., i_{k-1}=1}^{N} W_{k}\left(x_{i_{1}}, x_{i_{1}}, x_{i_{2}} . ., x_{i_{k-1}}\right) .
$$

Then,

$$
\frac{1}{2} \int\left(W_{2}-W_{\epsilon}\right) d\left(\hat{\mu}^{N}\right)^{\otimes 2}+\sum_{k=3}^{r} \frac{1}{k !} \int W_{k} d\left(\hat{\mu}^{N}\right)^{\otimes k}=\sum_{k=2}^{r} U_{N}^{k}(X)+Z_{N}(X) .
$$


Therefore, Cauchy-Schwartz inequality yields

$$
R_{N}(\gamma, \epsilon) \leq \mathbb{E}\left[\exp \left\{2 N \gamma \sum_{k=2}^{r} U_{N}^{k}(X)\right\}\right]^{\frac{1}{2}} \mathbb{E}\left[\exp \left\{2 N \gamma Z_{N}(X)\right\}\right]^{\frac{1}{2}}
$$

Let us first focus on

$$
R_{N}^{1}(\gamma, \epsilon):=\mathbb{E}\left[\exp \left\{2 N \gamma \sum_{k=2}^{r} U_{N}^{k}(X)\right\}\right] .
$$

Applying Cauchy-Schwartz's inequality $r-2$ times, we get

$$
R_{N}^{1}(\gamma, \epsilon) \leq \prod_{k=2}^{r} \mathbb{E}\left[\exp \left\{N 2^{k} \gamma U_{N}^{k}(X)\right\}\right]^{\frac{1}{2^{k}}}
$$

According to Lemma 3.1, for $N \geq N_{0}$ large enough, as soon as $\int\left(W_{2}-W_{\epsilon}\right)^{2} d\left(\mu^{*}\right)^{\otimes 2}$ is small enough (depending on $\gamma$ ), there exists a finite constant $c$ such that

$$
\mathbb{E}\left[\exp \left\{2 N \gamma U_{N}^{2}(X)\right\}\right] \leq c .
$$

For the other terms in the r.h.s of (29), as well for $N$ large enough,

$$
\mathbb{E}\left[\exp \left\{2^{k-1} N \gamma U_{N}^{k}(X)\right\}\right] \leq 2
$$

in view of Lemma 3.2. Therefore, for $\epsilon$ small enough and $N$ large enough,

$$
R_{N}^{1}(\gamma, \epsilon) \leq 2^{\frac{1}{4}} c^{\frac{1}{2}}
$$

Let us now focus on

$$
R_{N}^{2}(\gamma, \epsilon):=\mathbb{E}\left[\exp \left\{2 N \gamma Z_{N}(X)\right\}\right] .
$$

This part is actually on the scale of the law of large numbers. In fact, if the $\left(W_{k}\right)_{k \geq 2}$ were continuous, $N Z_{N}$ would be a bounded continuous function of the empirical measure. Hence, the law of large numbers would imply that

$$
\lim _{N \rightarrow \infty} N Z_{N}=\lim _{N \rightarrow \infty} N \mathbb{E}\left[Z_{N}\right]=\frac{1}{2} \int\left(W_{2}-W_{\epsilon}\right)(x, x) d \mu^{*}(x) \quad \text { a.s. }
$$

Thus, dominated convergence theorem would end the proof of the lemma. To avoid the previous assumption, we shall decompose $Z_{N}$ in terms of $U$-statistics and use again our controls on $U$-statistics. Indeed, denoting $\widetilde{W}_{k}\left(x_{i_{1}} . ., x_{i_{k-2}}\right)$ (resp. $\left.\bar{W}_{k}\left(x_{i_{1}} . ., x_{i_{k-1}}\right)\right)$ for $\int W_{k}\left(x, x, x_{i_{1}} . ., x_{i_{k-2}}\right) d \mu^{*}(x)$ (resp. for the symmetric version of $\left.W_{k}\left(x_{i_{1}}, x_{i_{1}}, x_{i_{2}} . ., x_{i_{k-1}}\right)-\widetilde{W}_{k}\left(x_{i_{1}} . ., x_{i_{k-2}}\right)\right)$, one checks that

$$
\begin{aligned}
N Z_{N}(X)= & \frac{1}{2} \int\left(W_{\epsilon}-W_{2}\right)(x, x) d \mu^{*}(x)+\frac{1}{2} \int\left(\left(W_{2}-W_{\epsilon}\right)(x, x)+\widetilde{W}_{3}(x)\right) d\left(\hat{\mu}^{N}-\mu^{*}\right)(x) \\
& +\frac{1}{2} \sum_{k=4}^{r} \frac{1}{N^{k-2}} \sum_{i_{1}<. .<i_{k-2}} \widetilde{W}_{k}\left(x_{i_{1}}, x_{i_{2} . . .}, x_{i_{k-2}}\right) \\
& +\frac{1}{2} \sum_{k=3}^{r} \frac{k-1}{N^{k-1}} \sum_{i_{1}<. .<i_{k-1}} \bar{W}_{k}\left(x_{i_{1}}, x_{i_{2}} . ., x_{i_{k-1}}\right)+O\left(\frac{1}{N}\right) .
\end{aligned}
$$


Therefore, we find a finite constant $C$ so that

$$
\begin{aligned}
R_{N}^{2}(\gamma, \epsilon) \leq & \exp \left\{\frac{C \gamma}{N}+\gamma \int\left(W_{\epsilon}-W_{2}\right)(x, x) d \mu^{*}(x)\right\} \\
& \times \mathbb{E}\left[\exp \left\{2 \gamma \int\left(W_{2}-W_{\epsilon}+\widetilde{W}_{3}\right)(x, x) d\left(\hat{\mu}^{N}-\mu^{*}\right)(x)\right\}\right]^{\frac{1}{2}} \\
& \times \mathbb{E}\left[\exp 2 \gamma\left\{\sum_{k=4}^{r} \frac{1}{N^{k-2}} \sum_{i_{1}<. .<i_{k-2}} \widetilde{W}_{k}\left(x_{i_{1}}, . ., x_{i_{k-2}}\right)+\sum_{k=3}^{r} \frac{k-1}{N^{k-1}} \sum_{i_{1}<. .<i_{k-1}} \bar{W}_{k}\left(x_{i_{1}}, . ., x_{i_{k-1}}\right)\right\}\right]^{\frac{1}{2}} .
\end{aligned}
$$

Since $\widetilde{W}_{k}$ and $\bar{W}_{k}$ satisfy the hypotheses of Lemmas 3.1 and 3.2 , we see that there exists a finite constant $C=C(\gamma)$ so that the last term in the r.h.s. of (33) is bounded by $e^{\frac{C}{N}}$ for $N$ large enough. For the second term, one can compute it and get a similar bound. Thus, for large enough $N$, we get an upper bound of the form

$$
R_{N}^{2}(\gamma, \epsilon) \leq e^{\gamma \int\left(W_{\epsilon}-W_{2}\right) d \mu^{*}} e^{\frac{C}{N}} .
$$

This gives Lemma 2.6 with (28-30) and (31). Note that in fact, since $N Z_{N}$ is bounded, it was enough to control the convergence in probability of $N Z_{N}$ to get our result, and for instance its convergence in $L^{2}\left(\left(\mu^{*}\right)^{\otimes \infty}\right)$. However, because we already have the above controls on $U$-statistics, we chose to use them.

Lemmas 2.5 and 2.6 with inequality (26) give inequality (25), thus Lemma 2.4a). Let us now turn to the lower bound Lemma 2.4b) and show that for any $\eta>0$, any $A>0$, and for $\alpha>1$ small enough, any $\delta>0$ and $\epsilon>0$ small enough (depending on $A$ and $\alpha$ )

$$
\begin{aligned}
\liminf _{N \rightarrow \infty} \widetilde{Z}_{N}^{F, f} & \geq e^{-\eta} \frac{1}{\sqrt{2 \pi \operatorname{det}\left(I-\Xi_{\epsilon}\right) \sigma_{\epsilon}(f)}} \int F(x) e^{-\frac{x^{2}}{2 \sigma_{\epsilon}(f)}} d x \\
& -e^{-\eta A} e^{(A / 2) \int\left(W_{2}-W_{\varepsilon}\right)(x, x) d \mu^{*}(x)}\|F\|_{\infty} \operatorname{det}\left(I-\alpha \Xi_{\epsilon}\right)^{-\frac{1}{2 \alpha}} .
\end{aligned}
$$

To this end, let us write for any $\eta>0$

$$
\begin{aligned}
\widetilde{Z}_{N}^{\delta, \epsilon} & \geq \mathbb{E}\left[\mathbb{I}_{B_{\delta}^{\epsilon}\left(\hat{\mu}^{N}\right) \cap\left\{N\left|\widetilde{\Gamma}\left(\hat{\mu}^{N}\right)-\Gamma_{\varepsilon}\left(\hat{\mu}^{N}\right)\right| \leq \eta\right\}} F e^{\left.N \widetilde{\Gamma}\left(\hat{\mu}^{N}\right)\right]}\right. \\
& \geq e^{-\eta} \mathbb{E}\left[\mathbb{I}_{B_{\delta}^{\epsilon}\left(\hat{\mu}^{N}\right)} F \exp \left\{N \Gamma_{\varepsilon}\left(\hat{\mu}^{N}\right)\right\}\right]-e^{-\eta}\|F\|_{\infty} \mathbb{E}\left[\mathbb{I}_{A_{N}(\delta, \eta)} \exp \left\{N \Gamma_{\epsilon}\left(\hat{\mu}^{N}\right)\right\}\right]
\end{aligned}
$$

with

Set

$$
A_{N}(\delta, \eta)=B_{\delta}^{\epsilon}\left(\hat{\mu}^{N}\right) \cap\left\{N \mid \widetilde{\Gamma}\left(\hat{\mu}^{N}\right)-\Gamma_{\varepsilon}\left(\hat{\mu}^{N}\right) \geq \eta\right\}
$$

$$
\begin{aligned}
I_{N}^{1}(\eta, \epsilon, \delta) & =e^{-\eta} \mathbb{E}\left[\mathbb{I}_{B_{\delta}^{\epsilon}\left(\hat{\mu}^{N}\right)} F \exp \left\{N \Gamma_{\varepsilon}\left(\hat{\mu}^{N}\right)\right\}\right] \\
I_{N}^{2}(\eta, \epsilon, \delta) & =e^{-\eta} \mathbb{E}\left[\mathbb{I}_{A_{N}(\delta, \eta)} \exp \left\{N \Gamma_{\epsilon}\left(\hat{\mu}^{N}\right)\right\}\right] .
\end{aligned}
$$

Let us first notice that, for any positive $\delta$ small enough,

$$
\lim _{N \rightarrow \infty} I_{N}^{1}(\eta, \epsilon, \delta)=e^{-\eta} \frac{1}{\sqrt{2 \pi \operatorname{det}\left(I-\Xi_{\epsilon}\right) \sigma_{\epsilon}(f)}} \int F(x) e^{-\frac{x^{2}}{2 \sigma_{\epsilon}(f)}} d x
$$


according to our hypotheses and the now standard results of [3] and [12] (see [12], Lem. 3.2 for instance). Moreover, we also find that, if $\alpha>1$ is small enough as before and letting $\gamma$ be its conjugate exponent, Hölder and Chebyshev inequalities yield, for any real number $A>0$,

$$
I_{N}^{2}(\eta, \epsilon, \delta) \leq e^{-\eta A} \mathbb{E}\left[e^{N \alpha \Gamma_{\epsilon}\left(\hat{\mu}^{N}\right)}\right]^{\frac{1}{\alpha}} \mathbb{E}\left[e^{N \gamma A\left(\widetilde{\Gamma}-\Gamma_{\epsilon}\right)\left(\hat{\mu}^{N}\right)}\right]^{\frac{1}{\gamma}} .
$$

Lemma 2.5 shows that the first term in the above r.h.s is converging whereas Lemma 2.6 shows that the second term is bounded for any $\gamma A$ as soon as $\epsilon$ is small enough, depending on $\gamma A$. This shows that for any $\alpha>1$ small enough and any $A>0$, there exists $\epsilon(\alpha, A)>0$ so that for any $\epsilon \in(0, \epsilon(\alpha, A))$,

$$
\limsup _{N \rightarrow \infty} I_{N}^{2}(\eta, \epsilon, \delta) \leq e^{-\eta A} e^{(A / 2) \int\left(W_{2}-W_{\epsilon}\right)(x, x) d \mu^{*}(x)} \operatorname{det}\left(I-\alpha \Xi_{\epsilon}\right)^{\frac{-1}{2 \alpha}}
$$

(36) and (39) give equation (35).

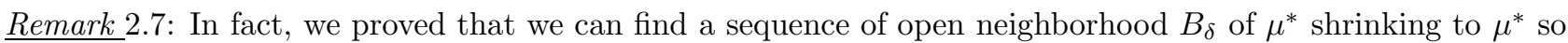
that for any bounded continuous function $F$ and any $f \in L_{0}^{2}\left(\mu^{*}\right)$,

$$
\lim _{\delta \downarrow 0} \lim _{p \downarrow 1} \lim _{N \rightarrow \infty} \frac{\int_{B_{\delta}} F\left(\frac{1}{\sqrt{N}} \sum f\left(x_{i}\right)\right) e^{N p \widetilde{\Gamma}\left(\hat{\mu}^{N}\right)} d P^{\otimes N}(X)}{\left(\int_{B_{\delta}} e^{N \widetilde{\Gamma}\left(\hat{\mu}^{N}\right)} d P^{\otimes N}(X)\right)^{p}}=\frac{1}{\sqrt{2 \pi \sigma(f)}} \int F(x) e^{-\frac{x^{2}}{2 \sigma(f)}} d x .
$$

\section{Estimates}

In this section, we get crucial upper bounds on exponential moments of $U$-statistics. These estimates are based on the works of De La Peña [15] and Arcones-Gine [1]. We first study the two-body setting and denote

$$
U_{N}^{2}(X):=\frac{1}{N^{2}} \sum_{1 \leq i<j \leq N} W\left(x_{i}, x_{j}\right) .
$$

Without loss of generality, we will assume that $W$ is symmetric and $\mu^{*}$-canonical. Let us denote

$$
\sigma^{2}:=\int W^{2}(x, y) d \mu^{*}(x) d \mu^{*}(y) .
$$

Our result states as follows

Lemma 3.1. If $W$ is regular, for any real number $\gamma$, there exists $\sigma(\gamma)>0$ so that, if $\sigma<\sigma(\gamma)$,

$$
\sup _{N \geq 0} \int \exp \left\{\gamma N U_{N}^{2}(X)\right\} d\left(\mu^{*}\right)^{\otimes N}(X)
$$

is finite.

Proof. Note in short

$$
R_{N}(\gamma, \delta):=\mathbb{E}\left[\exp \left\{\frac{\gamma}{N} \sum_{i<j} W\left(x_{i}, x_{j}\right)\right\}\right] .
$$

According to De La Peña [15] (see Th. 1 and Cor. 1) (see also Arcones-Gine [1], Th. 2.1, or the appendix), there exists universal constants $C_{0}$ and $C_{0}^{\prime}$ so that the following randomization inequality holds 


$$
\mathbb{E}\left[\exp \left\{\frac{\gamma}{N} \sum_{i<j} W\left(x_{i}, x_{j}\right)\right\}\right] \leq \mathbb{E}\left[\exp \left\{\frac{C_{0} \gamma}{N}\left|\sum_{i<j} \epsilon_{i} \epsilon_{j} W\left(x_{i}, x_{j}\right)\right|\right\}\right]^{C_{0}^{\prime}}
$$

where the $\epsilon_{i}$ are Rademacher's variables independent of the $x_{i}$ 's. Note

$$
\sigma_{N}^{2}(X):=\frac{1}{N^{2}} \sum_{i \neq j} W\left(x_{i}, x_{j}\right)^{2} .
$$

$\sigma_{N}$ converges $\left(\mu^{*}\right)^{\otimes N}$ almost surely to $\sigma$ according to the law of large numbers. The following decomposition holds

$$
\begin{aligned}
\mathbb{E}\left[\exp \left\{\frac{C_{0} \gamma}{N}\left|\sum_{i<j} \epsilon_{i} \epsilon_{j} W\left(x_{i}, x_{j}\right)\right|\right\}\right] & \leq \mathbb{E}\left[\mathbb{I}_{\sigma_{N}(X) \geq 2 \sigma} \exp \left\{\frac{C_{0} \gamma}{N}\left|\sum_{i<j} \epsilon_{i} \epsilon_{j} W\left(x_{i}, x_{j}\right)\right|\right\}\right] \\
& +\mathbb{E}\left[\exp \left\{2 \frac{C_{0} \gamma}{N}\left|\sigma \frac{\sum_{i<j} \epsilon_{i} \epsilon_{j} W\left(x_{i}, x_{j}\right)}{\sigma_{N}(X)}\right|\right\}\right] .
\end{aligned}
$$

To bound the first term in the r.h.s. of (42), note that

$$
\begin{aligned}
\mathbb{E}\left[\mathbb{I}_{\sigma_{N}(X) \geq 2 \sigma} \exp \left\{\frac{C_{0} \gamma}{N}\left|\sum_{i<j} \epsilon_{i} \epsilon_{j} W\left(x_{i}, x_{j}\right)\right|\right\}\right] & \leq \mathbb{E}\left[\mathbb{I}_{\sigma_{N}(X) \geq 2 \sigma} \exp \left\{\frac{C_{0} \gamma}{N} \sum_{i<j} \epsilon_{i} \epsilon_{j} W\left(x_{i}, x_{j}\right)\right\}\right] \\
& +\mathbb{E}\left[\mathbb{I}_{\sigma_{N}(X) \geq 2 \sigma} \exp \left\{-\frac{C_{0} \gamma}{N} \sum_{i<j} \epsilon_{i} \epsilon_{j} W\left(x_{i}, x_{j}\right)\right\}\right]
\end{aligned}
$$

so that we can neglect the absolute values in the following estimates to bound the r.h.s. of (42) since they will not depend on the sign of $W$.

Moreover, if $B$ denotes the Bernoulli law, $B(\epsilon)=(1 / 2) \delta_{\epsilon=+1}+(1 / 2) \delta_{\epsilon=-1}$, Sanov's theorem (see [9], Th. 6.2.10) shows that the empirical measure $(1 / N) \sum \delta_{x_{i}, \epsilon_{i}}$ satisfies a large deviation principle for the strong topology under $\left(\mu^{*} \otimes B\right)^{\otimes N}$ with good rate function the relative entropy $I\left(\mid \mu^{*} \otimes B\right)$. Since we assumed $\psi_{W}^{2}$ continuous for the strong topology, $\left\{\sigma_{N}(X) \geq 2 \sigma\right\}$ is a closed subset of $\mathcal{P}(\Sigma \times\{-1,+1\})$ furnished with the strong topology. Also, if $\psi_{W}^{1}$ is continuous, Laplace's method (see [9], Th. 4.3.1) implies

$$
\begin{aligned}
& \limsup _{N \rightarrow \infty} \frac{1}{N} \log \mathbb{E}\left[\mathbb{I}_{\sigma_{N}(X) \geq 2 \sigma} \exp \left\{\frac{C_{0} \gamma}{N} \sum_{i<j} \epsilon_{i} \epsilon_{j} W\left(x_{i}, x_{j}\right)\right\}\right] \\
& \leq-\inf \left\{I\left(\mu \mid \mu^{*} \otimes B\right)-(1 / 2) C_{0} \gamma \int W(x, y) \epsilon \widetilde{\epsilon} d \mu(x, \epsilon) d \mu(y, \widetilde{\epsilon})\right. \\
& \left.\int W^{2}(x, y) d \mu^{\otimes 2}(x, y) \geq 4 \int W^{2}(x, y) d\left(\mu^{*}\right)^{\otimes 2}(x, y)\right\} .
\end{aligned}
$$

To see that the infimum in the r.h.s. of (43) is negative, let us consider the function on $\mathcal{P}(\Sigma \times\{-1,+1\})$

$$
\widetilde{H}(\mu)=I\left(\mu \mid \mu^{*} \otimes B\right)-(1 / 2) C_{0} \gamma \int W(x, y) \epsilon \widetilde{\epsilon} d \mu(x, \epsilon) d \mu(y, \widetilde{\epsilon}) .
$$


$\widetilde{H}$ can easily be seen to have compact level sets and to be bounded from below. Thus, it achieves its minimum value and the minimizers verify

$$
\log \frac{d \mu}{d \mu^{*} \otimes B}(\epsilon, x)=C_{0} \gamma \epsilon \int W(x, y) \widetilde{\epsilon} d \mu(\widetilde{\epsilon}, y)+\text { const. }
$$

In particular, there exists a finite constant $M(\gamma)$ so that, for any minimizer $\mu$,

$$
\left\|\log \frac{d \mu}{d \mu^{*} \otimes B}\right\|_{\infty} \leq M(\gamma)
$$

Together with the inequality

$$
x \log x \geq \frac{3}{4+2 x}(x-1)^{2}+x-1,
$$

we see that there exists a positive constant $c(\gamma)$ so that, for any minimizing measure $\mu$ with density $f$ with respect to $\mu^{*} \otimes B$

$$
I\left(\mu \mid \mu^{*} \otimes B\right) \geq c(\gamma) \int(f-1)^{2} d \mu^{*} \otimes B .
$$

On the other hand, since $W$ is centered, Cauchy-Schwartz inequality yields

$$
\left|\int W \epsilon \widetilde{\epsilon} d \mu d \mu\right| \leq \sigma \int(f-1)^{2} d \mu^{*} \otimes B
$$

so that

$$
\widetilde{H}(\mu) \geq\left(c(\gamma)-C_{0} \gamma \sigma\right) \int(f-1)^{2} d \mu^{*} \otimes B
$$

Hence, for $\sigma$ small enough, $\widetilde{H}$ achieves its minimal value at $\mu^{*} \otimes B$. Its minimum value is thus zero and we deduce that the infimum in (43) is strictly positive for small positive $\sigma$ (but decreasing to zero with $\sigma$ ). As a consequence,

$$
\limsup _{N \rightarrow \infty} \frac{1}{N} \log \mathbb{E}\left[\mathbb{I}_{\sigma_{N}(X) \geq 2 \sigma} \exp \left\{\frac{C_{0} \gamma}{N} \sum_{i<j} \epsilon_{i} \epsilon_{j} W\left(x_{i}, x_{j}\right)\right\}\right]<0
$$

Finally, to bound the second term in the r.h.s. of (42), we follow Arcones-Gine who, using a result from Borel [6] (see (47)), found (see the proof of Prop. 2.3c) of [1]) that

$$
\sup _{N} \mathbb{E}\left[\exp \left\{2 \frac{C_{0} \gamma}{N} \sigma\left|\frac{\sum_{i<j} \epsilon_{i} \epsilon_{j} W\left(x_{i}, x_{j}\right)}{\sigma_{N}(X)}\right|\right\}\right]<\infty
$$

as soon as $C_{0} \gamma \sigma<(1 / e)$. This is true for $\sigma<\sigma(\gamma)$ with $\sigma(\gamma):=\left(1 / e C_{0} \gamma\right)$. (41), (44) and (45) give Lemma 2.5.

We now consider the exponential moments of more general $U$-processes. Let $m$ be an integer number, $m \geq 3$, and denote

$$
U_{N}^{m}(X):=\frac{1}{N^{m}} \sum_{1 \leq i_{1}<. .<i_{m} \leq N} W\left(x_{i_{1}}, . ., x_{i_{m}}\right)
$$

where $W$ is a symmetric bounded $\mu^{*}$-canonical function.

The next lemma shows that the exponential moments of $U_{N}^{m}$ exist for $N$ large enough (depending on $\|W\|_{\infty}$ and $m$ ). It is rather remarkable that this lemma allows us to neglect the derivatives of $\Gamma$ of order greater 
than two. One should compare it to the use of Yurinskii's lemma by Bolthausen in [5] for the same kind of approximation.

Lemma 3.2. Let $m \geq 3$ and $W$ be a bounded $\mu^{*}$-canonical on $\Sigma^{m}$. For any positive real number $\gamma$, if we denote

$$
\eta:=2^{\frac{3 m-2}{2}}(\sqrt{m !})^{-1}\|W\|_{\infty} \gamma
$$

for any integer number $N$ so that

$$
\log \frac{N}{2 \eta} \geq 2^{\frac{3 m-2}{2}} \eta \gamma\|W\|_{\infty}+\log \left(2^{\frac{3 m-2}{2}} \gamma e\|W\|_{\infty}\right), \quad \int \exp \left\{\gamma N U_{N}^{m}(X)\right\} d\left(\mu^{*}\right)^{\otimes N}(X) \leq 2 .
$$

Proof. Note in short

$$
R_{N}(\gamma):=\mathbb{E}\left[\exp \left\{\frac{\gamma}{N^{m-1}} \sum_{i_{1}<. .<i_{m}} W\left(x_{i_{1}}, . ., x_{i_{m}}\right)\right\}\right] .
$$

Again, we can use the decoupling and randomization techniques proposed by De la Peña [15] to find that the first term in the r.h.s. of (46) is bounded by

$$
V_{N}(\gamma):=\mathbb{E}\left[\exp \left(\frac{C_{0} \gamma}{N^{m-1}} \sum_{i_{1}<. .<i_{m}} \epsilon_{i_{1}}^{1} . . \epsilon_{i_{m}}^{m} W\left(x_{i_{1}}^{1}, . ., x_{i_{m}}^{m}\right)\right)\right]^{C_{0}^{\prime}}
$$

for some universal constants $C_{0}$ and $C_{0}^{\prime}$, independent Rademacher variables $\epsilon_{i}^{j}$,s, and independent copies $x_{i}^{j}$,s of the $x_{i}$ 's independent of the Rademacher variables. $C_{0}$ and $C_{0}^{\prime}$ depends on $m$ a priori. This result is proved in the appendix as well as bounds on these constants.

Recall the following result due to Borel [6] (see also [1], Prop. 2.2) which yields, for any integer number $q \geq 2$ and for any sequence $a_{i_{1}, . ., i_{m}}$ of real numbers

$$
\mathbb{E}\left[\left(\sum_{i_{1}<. .<i_{m}} \epsilon_{i_{1}}^{1} . . \epsilon_{i_{m}}^{m} a_{i_{1}, . ., i_{m}}\right)^{q}\right]^{\frac{1}{q}} \leq(q-1)^{\frac{m}{2}}\left(\sum_{i_{1}<. .<i_{m}} a_{i_{1}, . ., i_{m}}^{2}\right)^{\frac{1}{2}}
$$

where equality holds if $q=2$. Thus, if we let

$$
\widetilde{U}_{N}^{m}(X):=\frac{C_{0} \gamma}{N^{m-1}} \sum_{i_{1}<. .<i_{m}} \epsilon_{i_{1}}^{1} . . \epsilon_{i_{m}}^{m} W\left(x_{i_{1}}^{1}, . ., x_{i_{m}}^{m}\right)
$$

we find, conditionning by $X$ and using (47), that

$$
\mathbb{E}\left[\left(\widetilde{U}_{N}^{m}(X)\right)^{q}\right] \leq(q-1)^{q}\left(C_{0} \gamma\|W\|_{\infty}(\sqrt{m !})^{-1}\left(\frac{q-1}{N}\right)^{\frac{m-2}{2}}\right)^{q} .
$$

Moreover, Taylor expansion yields, for any $\epsilon>0$,

$$
\begin{aligned}
V_{N}(\gamma)= & \sum_{q=0}^{[\epsilon N]} \frac{1}{2 q !} \mathbb{E}\left[\left(\widetilde{U}_{N}^{m}(X)\right)^{2 q}\right]+\frac{1}{2[\epsilon N] !}\left(C_{0} \gamma\|W\|_{\infty}\right)^{2[\epsilon N]} \exp \left(C_{0} \gamma\|W\|_{\infty} N\right) \\
& \leq \frac{1}{1-\left(2 e C_{0} \gamma(\sqrt{m !})^{-1}\|W\|_{\infty} \epsilon\right)^{2}}+\frac{1}{[\epsilon N] !}\left(C_{0} \gamma\|W\|_{\infty}\right)^{2[\epsilon N]} \exp \left(C_{0} \gamma\|W\|_{\infty} N\right)
\end{aligned}
$$


where we have used in the last line (48) and Stirling's formula. We have also assumed $\epsilon$ small enough to insure that $2 e C_{0} \gamma(\sqrt{m !})^{-1}\|W\|_{\infty} \epsilon<1$. Using the bound on $C_{0}$ provided in Lemma 5.1 , we can take $\epsilon=(1 / 2 \eta)$. It is then straightforward to check that the first term is bounded by $(4 / 3)$ whereas the second term is going to zero as $N$ goes to infinity. Therefore, since $C_{0}^{\prime}$ is smaller than one, $V_{N}(\gamma)$ is smaller than 2 for $N$ large enough and the proof is complete.

\section{Generalization: The infinitely many Body Setting}

In this section, we wish to consider the case where $\Gamma(\mu)$ is not a polynomial function of $\mu$ but can be written

$$
\Gamma(\mu)=\sum_{k=0}^{\infty} \frac{1}{k !} \int W_{k}\left(x_{1}, . ., x_{k}\right) d(\mu)^{\otimes k}\left(x_{1}, . ., x_{k}\right)
$$

with $\mu^{*}$-canonical bounded functions $\left(W_{k}\right)_{k \geq 0}$. $\Gamma$ is somehow analytic for the Frechet derivation $D$. Furthermore, we will assume that

(H2) There exists a finite constant $C$ such that for any $k \in \mathbb{N}$,

$$
\left\|W_{k}\right\|_{\infty} \leq C c^{k} \sqrt{k !}
$$

where $c$ is a universal constant.

Proposition 4.1. Assume that (HO), (H1) and (H2) hold, and that $W_{2}$ is bounded regular. Then, for any bounded continuous function $F$ and any $f \in L_{0}^{2}\left(\mu^{*}\right)$,

$$
\lim _{N \rightarrow \infty} \int F\left(\frac{1}{\sqrt{N}} \sum_{i=1}^{N} f\left(x_{i}\right)\right) d P_{\Gamma}^{N}=\lim _{\epsilon \downarrow 0} \lim _{N \rightarrow \infty} \int F\left(\frac{1}{\sqrt{N}} \sum_{i=1}^{N} f\left(x_{i}\right)\right) d P_{\Gamma_{\epsilon}}^{N} .
$$

As a consequence, $\frac{1}{\sqrt{N}} \sum_{i=1}^{N} f\left(x_{i}\right)$ converges under $P_{\Gamma}^{N}$ towards a centered Gaussian variable with covariance $\sigma(f)$.

Proof. First, let us notice that, as before, for any bounded measurable function $F$, the partition function $\int F \exp \left\{N \widetilde{\Gamma}\left(\hat{\mu}^{N}\right)\right\} d\left(\mu^{*}\right)^{\otimes N}$ is equivalent, up to an exponentially small term, for any open neighborhood $B\left(\mu^{*}\right)$ of $\mu^{*}$ for the strong topology, to

$$
\widetilde{Z}_{N}(F):=\int \mathbb{I}_{B\left(\mu^{*}\right)} F \exp \left\{N \widetilde{\Gamma}\left(\hat{\mu}^{N}\right)\right\} d\left(\mu^{*}\right)^{\otimes N}
$$

In the following, we shall be given a bounded non negative continuous function $F$ on $\mathbb{R}$ and $f \in L_{0}^{2}\left(\mu^{*}\right)$. Moreover, denote for a fixed $m$

$$
\Gamma_{m}(\mu)=\sum_{k=2}^{m} \frac{1}{k !} \int W_{k} d \mu^{\otimes k}
$$

and write

$$
\widetilde{\Gamma}\left(\hat{\mu}^{N}\right)=\Gamma\left(\hat{\mu}^{N}\right)-\Gamma\left(\mu^{*}\right)-D \Gamma\left[\mu^{*}\right]\left(\hat{\mu}^{N}-\mu^{*}\right)=\Gamma_{m}\left(\hat{\mu}^{N}\right)+R_{m}\left(\hat{\mu}^{N}\right) .
$$

By Hölder's inequality, for any $p>1$ with conjugate exponent $q$

$$
\widetilde{Z}_{N}(F) \leq\left(\int \mathbb{I}_{B\left(\mu^{*}\right)} F^{p} \exp \left\{N p \Gamma_{m}\left(\hat{\mu}^{N}\right)\right\} d\left(\mu^{*}\right)^{\otimes N}\right)^{\frac{1}{p}}\left(\int \exp \left\{N q R_{m}\left(\hat{\mu}^{N}\right)\right\} d\left(\mu^{*}\right)^{\otimes N}\right)^{\frac{1}{q}} .
$$


Moreover, for any $\eta>0$,

$$
\begin{aligned}
\widetilde{Z}_{N}(\mathbb{I}) & \geq e^{-\eta} \int e^{N \Gamma_{m}\left(\hat{\mu}^{N}\right)} \mathbb{I}_{B\left(\mu^{*}\right) \cap\left\{\left|N R_{m}\left(\hat{\mu}^{N}\right)\right| \leq \eta\right\}} d\left(\mu^{*}\right)^{\otimes N} \\
& \geq e^{-\eta} \int \mathbb{I}_{B\left(\mu^{*}\right)} e^{N \Gamma_{m}\left(\hat{\mu}^{N}\right)} d\left(\mu^{*}\right)^{\otimes N}-e^{-\eta} \int e^{N \Gamma_{m}\left(\hat{\mu}^{N}\right)} \mathbb{I}_{B\left(\mu^{*}\right) \cap\left\{\left|N R_{m}\left(\hat{\mu}^{N}\right)\right| \geq \eta\right\}} d\left(\mu^{*}\right)^{\otimes N} .
\end{aligned}
$$

Hence,

$$
\int F d P_{\Gamma}^{N}=\frac{\widetilde{Z}_{N}(F)}{\widetilde{Z}_{N}(\mathbb{I})} \leq I_{N}^{p}(F) J_{N} K_{N}^{p}
$$

with

$$
\begin{aligned}
I_{N}^{p}(F) & :=\left(\frac{\int \mathbb{I}_{B\left(\mu^{*}\right)} F^{p} \exp \left\{N p \Gamma_{m}\left(\hat{\mu}^{N}\right)\right\} d\left(\mu^{*}\right)^{\otimes N}}{\left(\int \mathbb{I}_{B\left(\mu^{*}\right)} \exp \left\{N \Gamma_{m}\left(\hat{\mu}^{N}\right)\right\} d\left(\mu^{*}\right)^{\otimes N}\right)^{p}}\right)^{\frac{1}{p}} \\
J_{N} & :=\left(e^{-\eta}-e^{-\eta} \frac{\int \mathbb{I}_{B\left(\mu^{*}\right) \cap\left\{\left|N R_{m}\left(\hat{\mu}^{N}\right)\right| \geq \eta\right\}} d\left(\mu^{*}\right)^{\otimes N}}{\int \mathbb{I}_{B\left(\mu^{*}\right)} e^{N \Gamma_{m}\left(\hat{\mu}^{N}\right)} d\left(\mu^{*}\right)^{\otimes N}}\right)^{-1} \\
K_{N}^{p} & :=\left(\int \exp \left\{N q R_{m}\left(\hat{\mu}^{N}\right)\right\} d\left(\mu^{*}\right)^{\otimes N}\right)^{\frac{1}{q}} .
\end{aligned}
$$

In view of Remark 2.7, for any $m \geq 2$, we can choose a sequence of open neighborhoods $B$ of $\left\{\mu^{*}\right\}$ so that

$$
\lim _{B \downarrow\left\{\mu^{*}\right\}} \lim _{p \downarrow 1} \lim _{N \rightarrow \infty} I_{N}^{p}=\frac{1}{\sqrt{2 \pi \sigma(f)}} \int F(x) e^{-\frac{x^{2}}{2 \sigma(f)}} d x .
$$

Let us now consider the last term in the r.h.s. of (52). We have, according to our assumption (H2) on the derivatives of $\Gamma$, for any integer number $M \geq m$,

$$
R_{m}\left(\hat{\mu}^{N}\right)=\sum_{k=m+1}^{M} \frac{1}{k !} \int W_{k} d(\mu)^{\otimes k}+0\left(\frac{c^{M}}{\sqrt{M !}}\right) .
$$

In the following, we will choose $M=M(N)$ so that:

$$
\lim _{N \rightarrow \infty} N \frac{c^{M}}{\sqrt{M !}}=0
$$

Thus, applying Hölder's inequality inductively, we find:

$$
\int \exp \left\{N q R_{m}\left(\hat{\mu}^{N}\right)\right\} d\left(\mu^{*}\right)^{\otimes N} \leq \exp \left\{q N 0\left(\frac{c^{M}}{\sqrt{M !}}\right)\right\} \prod_{k=m+1}^{M}\left(\int \exp \left\{\frac{N q 2^{k-m}}{k !} \int W_{k} d\left(\hat{\mu}^{N}\right)^{\otimes k}\right\} d\left(\mu^{*}\right)^{\otimes N}\right)^{\frac{1}{2^{k-m}}} .
$$


But

$$
\begin{aligned}
\int \exp & \left\{\frac{N q 2^{k-m}}{k !} \int W_{k} d\left(\hat{\mu}^{N}\right)^{\otimes k}\right\} d\left(\mu^{*}\right)^{\otimes N}=\int \exp \left\{\frac{N^{1-k} q 2^{k-m}}{k !} \sum_{p_{1}, . ., p_{k}} W_{k}\left(x_{p_{1}}, . ., x_{p_{k}}\right)\right\} d\left(\mu^{*}\right)^{\otimes N} \\
\leq & \left(\int \exp \left\{N^{1-k} q 2^{k-m+1} \sum_{p_{1}<. .<p_{k}} W_{k}\left(x_{p_{1}}, . ., x_{p_{k}}\right)\right\} d\left(\mu^{*}\right)^{\otimes N}\right)^{\frac{1}{2}} \\
& \times\left(\int \exp \left\{\frac{N^{1-k} q 2^{k-m}}{(k-2) !} \sum_{p_{1}, . ., p_{k-1}} W_{k}\left(x_{p_{1}}, x_{p_{1}}, . ., x_{p_{k}}\right)\right\} d\left(\mu^{*}\right)^{\otimes N}\right)^{\frac{1}{2}} .
\end{aligned}
$$

According to Lemma 3.2, we know that, as long as

$$
\eta(k):=q 2^{\frac{5}{2} k-m} \sqrt{k !}\left\|W_{k}\right\|_{\infty} \leq C q 2^{\frac{5}{2} k-m} c^{k}
$$

stays uniformly bounded by say $\eta$,

$$
\int \exp \left\{N^{1-k} q 2^{k-m+1} \sum_{p_{1}<. .<p_{k}} W_{k}\left(x_{p_{1}}, . ., x_{p_{k}}\right)\right\} d\left(\mu^{*}\right)^{\otimes N} \leq 2
$$

for any integer number $N$ larger than some $N(\eta)$. Since $\eta(k)$ goes to zero when $k$ goes to infinity as soon as we have chosen our universal constant $c<2^{-\frac{5}{2}}$, this bound always holds. The exponent in the second term in the r.h.s. of (55) is uniformly bounded by

$$
\frac{q 2^{k-m}}{(k-2) !}\left\|W_{k}\right\|_{\infty} \leq \frac{q 2^{k-m} c^{k} k}{\sqrt{(k-2) !}}
$$

and therefore goes uniformly to zero as $k$ goes to infinity.

Plugging these results into (54) shows that, for any $q$, for $m$ and $N$ large enough,

$$
\limsup _{N \rightarrow \infty} \int \exp \left\{N q R_{m}\left(\hat{\mu}^{N}\right)\right\} d\left(\mu^{*}\right)^{\otimes N} \leq 2 \exp \{q \epsilon(m)\}
$$

where $\epsilon(m)$ goes to zero when $m$ goes to infinity. Note that the same bound holds if one replaces $R_{m}$ by $-R_{m}$, and therefore $R_{m}$ by $\left|R_{m}\right|$ if the above constant 2 is replaced by 4 . Hence, for any $q$, for $m$ and $N$ large enough,

$$
K_{N}^{p} \leq 2^{\frac{1}{q}} \exp \{\epsilon(m)\}
$$

Further, for any $a>0$, Chebyshev inequality implies that

$$
\begin{array}{r}
\int \frac{\int \mathbb{I}_{B\left(\mu^{*}\right) \cap\left\{\left|N R_{m}\left(\hat{\mu}^{N}\right)\right| \geq \eta\right\}} e^{N \Gamma_{m}\left(\hat{\mu}^{N}\right)} d\left(\mu^{*}\right)^{\otimes N}}{\int \mathbb{I}_{B\left(\mu^{*}\right)} e^{N \Gamma_{m}\left(\hat{\mu}^{N}\right)} d\left(\mu^{*}\right)^{\otimes N}} \leq e^{-a \eta} \frac{\int \mathbb{I}_{B\left(\mu^{*}\right)} e^{N \Gamma_{m}\left(\hat{\mu}^{N}\right)+a N\left|R_{m}\right|\left(\hat{\mu}^{N}\right)} d\left(\mu^{*}\right)^{\otimes N}}{\int \mathbb{I}_{B\left(\mu^{*}\right)} e^{N \Gamma_{m}\left(\hat{\mu}^{N}\right)} d\left(\mu^{*}\right)^{\otimes N}} \\
\leq e^{-a \eta}\left(\frac{\int \mathbb{I}_{B\left(\mu^{*}\right)} e^{p N \Gamma_{m}\left(\hat{\mu}^{N}\right)} d\left(\mu^{*}\right)^{\otimes N}}{\left(\int \mathbb{I}_{B\left(\mu^{*}\right)} e^{N \Gamma_{m}\left(\hat{\mu}^{N}\right)} d\left(\mu^{*}\right)^{\otimes N}\right)^{p}}\right)^{\frac{1}{p}}\left(\int \mathbb{I}_{B\left(\mu^{*}\right)} e^{a q N\left|R_{m}\right|\left(\hat{\mu}^{N}\right)} d\left(\mu^{*}\right)^{\otimes N}\right)^{\frac{1}{q}}
\end{array}
$$


where $(p, q)$ are conjugate exponents as above. Hence (56) and (53) shows that for $p>1$ small enough and $N$ large enough,

$$
\frac{\int \mathbb{I}_{B\left(\mu^{*}\right) \cap\left\{\left|N R_{m}\left(\hat{\mu}^{N}\right)\right| \geq \eta\right\}} e^{N \Gamma_{m}\left(\hat{\mu}^{N}\right)} d\left(\mu^{*}\right)^{\otimes N}}{\int \mathbb{I}_{B\left(\mu^{*}\right)} e^{N \Gamma_{m}\left(\hat{\mu}^{N}\right)} d\left(\mu^{*}\right)^{\otimes N}} \leq 8 e^{-a \eta+a \epsilon(m)}
$$

Consequently, for $m, q$ and $N$ large enough

$$
J_{N}^{\delta, \epsilon} \leq\left(e^{-\eta}-2 e^{-\eta} e^{-a \eta+a \epsilon(m)}\right)^{-1}
$$

$(50,52,53,57,58)$ and $(59)$ show that, letting first $N$ going, then $p$ towards one, $m$ to infinity, $\eta$ towards zero and $B$ shrinking to $\left\{\mu^{*}\right\}$,

$$
\limsup _{N \rightarrow \infty} \int F\left(\frac{1}{\sqrt{N}} \sum_{i=1}^{N} f\left(x_{i}\right)\right) d P_{\Gamma}^{N} \leq \frac{1}{\sqrt{2 \pi \sigma(f)}} \int F(x) e^{-\frac{x^{2}}{2 \sigma(f)}} d x .
$$

The lower bound is obtained similarly. We leave the details to the reader.

\section{Appendix}

In the first part of this appendix, we sketch a few of the proofs of the results borrowed from [15] and [1]. Indeed, they are very simple and give explicit bounds on the constants encountered in this article. The main idea introduced by De la Peña is contained, as far as we are concerned, in the following lemma

Lemma 5.1. For any integer number $m \geq 1$, for any probability measure $\mu$ on $\Sigma$, for any bounded $\mu$-canonical function $W$ on $\Sigma^{m}$,

$$
\int e^{\sum_{1 \leq i_{1}<. .<i_{m} \leq N} W\left(x_{i_{1}}, . ., x_{i_{m}}\right)} \prod_{i=1}^{N} d \mu\left(x_{i}\right) \leq\left(\int e^{2^{m-1} \sum_{i_{1}<. .<i_{m}} W\left(x_{i_{1}}^{1}, \ldots, x_{i_{m}}^{m}\right)} \prod_{j=1}^{m} \prod_{i=1}^{N} d \mu\left(x_{i}^{j}\right)\right)^{2^{1-m}} .
$$

Proof. The idea of the proof is based on the observation that

$$
M_{N}(W):=\frac{\exp \left\{\sum_{1 \leq i_{1}<. .<i_{m} \leq N} W\left(x_{i_{1}}, . ., x_{i_{m}}\right)\right\}}{\prod_{k=1}^{N} \int \exp \left\{\sum_{1 \leq i_{1}<. .<i_{m} \leq k-1} W\left(x_{i_{1}}, . ., x_{i_{m-1}}, y\right)\right\} d \mu(y)}
$$

is a $\mu^{\otimes N}$-martingale for the filtration $\left\{\sigma\left(x_{j}, j \leq n\right), 1 \leq n \leq N\right\}$ with mean 1. Moreover, Cauchy-Schwartz inequality yields

$$
\begin{aligned}
& \int e^{\sum_{1 \leq i_{1}<. .<i_{m} \leq N} W\left(x_{i_{1}}, \ldots, x_{i_{m}}\right)} \prod_{i=1}^{N} d \mu\left(x_{i}\right) \leq\left(\int M_{N}(2 W) d \mu^{\otimes N}\right)^{\frac{1}{2}} \\
& \quad \times\left(\int \prod_{k=1}^{N} \int \exp \left\{2 \sum_{1 \leq i_{1}<. .<i_{m} \leq k-1} W\left(x_{i_{1}}, . ., x_{i_{m-1}}, y\right)\right\} d \mu(y) d \mu^{\otimes N}\right)^{\frac{1}{2}} .
\end{aligned}
$$

The first term in the r.h.s. of (60) is equal to one. For the second term, we can proceed by induction with given $y$ 's. We then find Lemma 5.1. 
The introduction of Rademacher's variables is then classical since we have

Lemma 5.2. For any probability measure $\mu$, any measurable function $W$ such that $\int W d \mu=0$, if $B=(1 / 2)$ $\left(\delta_{-1}+\delta_{+1}\right)$,

$$
\int e^{W(x)} d \mu(x) \leq \int e^{\sqrt{2} \epsilon W(x)} d \mu(x) d B(\epsilon) .
$$

Proof. Again the proof is straightforward since the statement is equivalent, via Taylor expansion and for centered functions $W$, to

$$
\int W^{2}(x) \int_{0}^{1}(1-t) e^{t W(x)} d t d \mu(x) \leq \int W^{2}(x) \int_{0}^{1}(1-t)\left(e^{\sqrt{2} t W(x)}+e^{-\sqrt{2} t W(x)}\right) d t d \mu(x)
$$

which is clear.

Therefore, applying this result to $U$-statistics yields

Lemma 5.3. For any integer number $m \geq 1$, for any bounded measurable function $W$ on $\S^{m}$, for any probability measure $\mu$ on $\Sigma$, if $\int W(x) d \mu\left(x_{i}\right)=0$ for $i \in[1, m]$,

$$
\int e^{\sum_{1 \leq i_{1}<.<i_{m} \leq N} W\left(x_{i_{1}}, . ., x_{i_{m}}\right)} \prod_{i=1}^{N} d \mu\left(x_{i}\right) \leq\left(\int e^{2^{\frac{3 m-2}{2}} \sum_{i_{1}<. .<i_{m}} \epsilon_{i_{1}}^{1} . . \epsilon_{i_{m}}^{m} W\left(x_{i_{1}}^{1}, . ., x_{i_{m}}^{m}\right)} \prod_{j=1}^{m} \prod_{i=1}^{N} d \mu\left(x_{i}^{j}\right)\right)^{2^{1-m}} .
$$

The proof is again straightforward since it boils down to apply Lemma 5.3 to the $m \times N$ independent variables contained in the r.h.s. of Lemma 5.1.

Let us notice that the proof of the reverse inequality (41) is more involved and given in [15] for general $U$ statistics. It relies on the symmetry of the underlying functions.

Finally, let us show that

Lemma 5.4. Any bounded measurable function $W_{2}$ satisfies (H1).

Proof. The proof follows Arcones and Gine ([2] p. 660) where it is proven that any measurable function $W_{2} \in L^{2}\left(\mu^{*} \otimes \mu^{*}\right)$ can be approximated in $L^{2}\left(\mu^{*} \otimes \mu^{*}\right)$ by functions $W_{\epsilon}$ of type (2) but with bounded measurable functions $g_{\epsilon}$. To verify that this result can be strengthened into (H1), let us adapt its proof. Without loss of generality, we can assume $W_{2}$ non negative, the general case follows by considering separately the positive and the negative part of $W_{2}$. Further, by tightness of $\mu^{*}$, we can assume without loss of generality that $\Sigma$ is compact. Finally, we take $\left\|W_{2}\right\|_{\infty}=1$ to simplify the notations.

We shall prove that for any $\epsilon>0$, we can find a continuous function $W_{2}^{\epsilon}$ bounded by one of the form $\sum_{i=1}^{s^{\epsilon}} c_{i}^{\epsilon} \psi_{i}^{\epsilon}(x) \psi_{i}^{\epsilon}(y)$ with continuous functions $\left(\psi_{i}^{\epsilon}\right)_{1 \leq i \leq s^{\epsilon}}$ bounded by one, a finite integer number $s^{\epsilon}$ and with constants $\left(c_{i}\right)_{1 \leq i \leq s^{\epsilon}}$ bounded by one satisfying

$$
\begin{aligned}
& \mu^{*} \otimes \mu^{*}\left(\left\{(x, y):\left|W_{2}-W_{2}^{\epsilon}\right|(x, y)>(\epsilon / 2)\right\}\right)<(\epsilon / 2) \\
& \text { and } \quad \mu^{*}\left(\left\{x:\left|W_{2}-W_{2}^{\epsilon}\right|(x, x)>(\epsilon / 2)\right\}\right)<(\epsilon / 2) .
\end{aligned}
$$

Clearly (61) and the boundedness of $W_{2}$ and $W_{2}^{\epsilon}$ implies (4) and (5). Moreover, if we consider the compact space $C=\left\{1, \ldots, s^{\epsilon}\right\}$ and the measure $\nu^{\varepsilon}\{i\}=c_{i}^{\varepsilon}$, then

$$
W_{2}^{\epsilon}\left(x_{1}, x_{2}\right)=\int \psi_{\tau}^{\varepsilon}\left(x_{1}\right) \psi_{\tau}^{\varepsilon}\left(x_{2}\right) d \nu(\tau)
$$


where $\psi_{\tau}($.$) is bounded continuous for every \tau$. Hence, $W_{2}^{\epsilon}$ satisfies the hypotheses of [3] and [12].

To prove (61), we shall first adapt Lusin's heorem (see Th. 2.24 of [16]) to show that there exists, for any $\epsilon>0$, a continuous function $W_{0}^{\epsilon}$ bounded by one so that

$$
\begin{aligned}
& \mu^{*} \otimes \mu^{*}\left(\left\{(x, y): W_{2}(x, y) \neq W_{0}^{\epsilon}(x, y)\right\}\right)<(\epsilon / 2) \\
& \text { and } \quad \mu^{*}\left(\left\{x: W_{2}(x, x) \neq W_{0}^{\epsilon}(x, x)\right\}\right)<(\epsilon / 2) .
\end{aligned}
$$

In fact, By tightness of $\mu^{*}$, we can assume without loss of generality that $\Sigma$ is compact. Thanks to Lusin's theorem we can construct two continuous functions $h^{\epsilon}$ on $\Sigma^{2}$ and $k^{\epsilon}$ on $\Sigma$ so that

$$
\mu^{*} \otimes \mu^{*}\left(\left\{(x, y): W_{2}(x, y) \neq h^{\epsilon}(x, y)\right\}\right)<(\epsilon / 6) \text { and } \mu^{*}\left(\left\{x: W_{2}(x, x) \neq k^{\epsilon}(x)\right\}\right)<(\epsilon / 6) .
$$

Let $\Delta=\{(x, x) ; x \in \Sigma\} \subset \Sigma^{2}$. Since $\Delta$ is a compact subset of $\Sigma^{2}$, Urysohn's lemma (see Lem. 2.12 in [16]) implies that we can find a continuous function $u^{\epsilon}$ bounded by one, so that $\left.u^{\epsilon}\right|_{\Delta}=1$ and

$$
\mu^{*} \otimes \mu^{*}\left(\left\{(x, y): u^{\epsilon}(x, y) \neq \mathbb{I}_{\Delta}(x, y)\right\}\right)<(\epsilon / 6)
$$

Set

$$
W_{0}^{\epsilon}(x, y)=u^{\epsilon}(x, y) k^{\epsilon}(x)+\left(1-u^{\epsilon}(x, y)\right) h^{\epsilon}(x, y) .
$$

Then, $W_{0}^{\epsilon}$ is continuous, bounded by one and

$$
\begin{aligned}
\mu^{*} \otimes \mu^{*}\left(\left\{(x, y): W_{2}(x, y) \neq W_{0}^{\epsilon}(x, y)\right\}\right) & \leq \mu^{*} \otimes \mu^{*}\left(\left\{(x, y): W_{2}(x, y) \neq h^{\epsilon}(x, y)\right\}\right) \\
& +\mu^{*} \otimes \mu^{*}\left(\left\{(x, y): x=y, W_{2}(x, x) \neq k^{\epsilon}(x)\right\}\right) \\
& +\mu^{*} \otimes \mu^{*}\left(\left\{(x, y): x \neq y, u^{\epsilon}(x, y) \neq 0\right\}\right) \\
& \leq(\epsilon / 2) .
\end{aligned}
$$

Also,

$$
\mu^{*}\left(\left\{(x): W_{2}(x, x) \neq W_{0}^{\epsilon}(x, x)\right\}\right)=\mu^{*}\left(\left\{(x): W_{2}(x, x) \neq k^{\epsilon}(x)\right\}\right)<(\epsilon / 6)
$$

which achieves the proof of (62).

Now, since we assumed $\Sigma$ compact, $W_{0}^{\epsilon}$ is uniformly continuous. Also, for any $\delta>0$, if $\left(A_{i}^{\delta}\right)_{1 \leq i \leq n^{\delta}}$ is a partition of $\Sigma$ of open sets of diameter less than $\delta$ (for a metric on the Polish space $\Sigma$ ) such that $A_{i}^{\delta} \cap A_{j}^{\delta}=\emptyset$ if $i \neq j, W_{1}^{\delta}(x, y)=\sum_{i, j=1}^{n^{\delta}} \inf _{A_{i}^{\delta} \times A_{j}^{\delta}} W_{0}^{\epsilon} \mathbb{I}_{A_{i}^{\delta}}(x) \mathbb{I}_{A_{j}^{\delta}}(y)$ converges point-wise towards $W_{0}^{\epsilon}$ as $\delta \downarrow 0$. Choose $\delta=\delta_{\epsilon}$ so that $\left\|W_{1}^{\delta_{\epsilon}}-W_{0}^{\epsilon}\right\|_{\infty}<(\epsilon / 2)$. $W_{1}^{\epsilon}$ is bounded by one since $A_{i}^{\delta} \cap A_{j}^{\delta}=\emptyset$ if $i \neq j$. Finally, by Urysohn's lemma, we approximate $\mathbb{I}_{A_{i}^{\delta_{\epsilon}}}$ by continuous functions $\phi_{i}^{\epsilon}$ bounded by one, null outside of $A_{i}^{\delta_{\epsilon}}$ and so that

$$
\mu^{*}\left(\left\{x: \phi_{i}^{\epsilon}(x) \neq \mathbb{I}_{A_{i}^{\delta_{\epsilon}}}(x)\right\}\right) \leq\left(\epsilon / 2^{i+1}\right) .
$$

In this way, we have constructed a continuous function $W_{2}^{\epsilon}$ bounded by one so that $W_{2}^{\epsilon}(x, y)=\sum_{i, j=1}^{n^{\delta_{\epsilon}}}\left(\inf _{A_{i}^{\delta_{\epsilon}} \times A_{j}^{\delta_{\epsilon}}}\right.$ $\left.W_{0}^{\epsilon}\right) \phi_{i}^{\epsilon}(x) \phi_{j}^{\epsilon}(y)$ and satisfying 


$$
\begin{aligned}
\mu^{*} \otimes \mu^{*}\left(\left\{(x, y): \mid W_{2}^{\epsilon}(x, y)\right.\right. & \left.\left.-W_{2}(x, y) \mid>(\epsilon / 2)\right\}\right) \leq \mu^{*} \otimes \mu^{*}\left(\left\{(x, y): W_{0}^{\epsilon}(x, y) \neq W_{2}(x, y)\right\}\right) \\
& +\mu^{*} \otimes \mu^{*}\left(\left\{(x, y):\left|W_{1}^{\epsilon}(x, y)-W_{0}^{\epsilon}(x, y)\right|>(\epsilon / 2)\right\}\right) \\
& +\mu^{*} \otimes \mu^{*}\left(\left\{(x, y): W_{1}^{\epsilon}(x, y) \neq W_{0}^{\epsilon}(x, y)\right\}\right) \\
& \leq \frac{\epsilon}{2}+\sum_{i=1}^{\delta_{\epsilon}} \mu^{*}\left(\left\{x: \phi_{i}^{\epsilon}(x) \neq \mathbb{I}_{A_{i}^{\delta_{\epsilon}}}(x)\right\}\right) \leq \epsilon .
\end{aligned}
$$

Similarly,

$$
\mu^{*} \otimes \mu^{*}\left(\left\{(x):\left|W_{2}^{\epsilon}(x, x)-W_{2}(x, x)\right|>(\epsilon / 2)\right\}\right) \leq \epsilon .
$$

Finally, if $W_{2}$ is symmetric, clearly $W_{0}^{\epsilon}$ can be chosen symmetric and therefore $W_{2}^{\epsilon}$. By polarisation, one then obtain, if $\left(\varepsilon_{1}, \varepsilon_{2}\right)$ are independent Bernoulli variables $P\left(\varepsilon_{1}=1\right)=P\left(\varepsilon_{2}=1\right)=P\left(\varepsilon_{1}=-1\right)=P\left(\varepsilon_{2}=-1\right)$ $=1 / 2$,

$$
W_{2}^{\epsilon}\left(x_{1}, x_{2}\right)=\frac{1}{2} \sum_{(i, j)=1}^{n^{\delta_{\epsilon}}} \inf _{A_{i}^{\delta} \times A_{j}^{\delta}} W_{0}^{\epsilon} E_{\varepsilon}\left[\varepsilon_{1} \varepsilon_{2}\left(\varepsilon_{1} \phi_{i}^{\epsilon}\left(x_{1}\right)+\varepsilon_{2} \phi_{j}^{\epsilon}\left(x_{1}\right)\right)\left(\varepsilon_{1} \phi_{i}^{\epsilon}\left(x_{2}\right)+\varepsilon_{2} \phi_{j}^{\epsilon}\left(x_{2}\right)\right)\right] .
$$

This is of the form announced at the beginning of the proof.

We wish to thank the Courant institute for welcoming us in 95-96 where we met by chance V. De la Peña who introduced us to his nice techniques. We also thank G. Ben Arous and J. Bretagnolle for encouraging discussions. We are very grateful to the referee whose comments allowed us to improve our results, in particular to weaken the hypotheses on $W_{2}$.

\section{REFERENCES}

[1] M.A. Arcones and E. Gine, Limit Theorems for U-processes. Ann. Probab. 21 (1993) 1494-1542.

[2] M.A. Arcones and E. Gine, On the bootstrap of $U$ and $V$ statistics. Ann. Stat. 20 (1992) 655-674.

[3] G. Ben Arous and M. Brunaud, Méthode de Laplace : Étude variationnelle des fluctuations de diffusions de type "champ moyen". Stochastics 31-32 (1990) 79-144.

[4] M.Sh. Birman, A proof of the Fredholm trace formula as an application of a simple embedding for kernels of integral operators of trace class in $L^{2}\left(\mathbb{R}^{m}\right)$. Lith-Mat-R-89-30 (1989).

[5] E. Bolthausen, Laplace approximation for sums of independent random vectors I. Prob. Th. Rel. Fields 72 (1986) $305-318$.

[6] C. Borell, On the integrability of Banach space valued Walsh polynomials. Séminaire de probabilités XIII. Lecture Notes in Math. $\mathbf{7 2 1}$ (1979) 1-3.

[7] D.A. Dawson, Critical dynamics and fluctuations for a mean field model of cooperative behavior. J. Stat. Phys. 31 (1983) 247-308.

[8] A. De Acosta and E. Gine, Convergence of moments and related Functionals in the central limit Theorem in Banach spaces. Z. Wahrsch. Verw. Gebiete 48 (1979) 213-231.

[9] A. Dembo and O. Zeitouni, Large deviations techniques and Applications. Jones and Bartlett (1992).

[10] J.D. Deuschel and D.W. Stroock, Large deviations. Academic press (1989).

[11] M. Hitsuda and H. Tanaka, Central limit Theorem for a simple diffusion model for interacting particles. Hiroshima Math. J. 11 (1981) 415-423.

[12] S. Kusuoka and Y. Tamura, Gibbs measures for mean field potentials. J. Fac. Sci. Univ. Tokyo Sect. IA Math. 31 (1984).

[13] H.P. Mac Kean, Fluctuations in the kinetic theory of gases. Comm. Pure Appl. Math. 28 (1975) 435-455.

[14] K.R. Parthasarathy, Probability measures on Metric Spaces. Academic Press Inc., New York (1968).

[15] V.H. De La Pena, Decoupling and Khintchine's inequalities for U-statistics. Ann. Probab. 20 (1992) 1877-1892.

[16] W. Rudin, Real and complex analysis, second edition, Springer.

[17] T. Shiga and H. Tanaka, Central limit Theorem for a system of markovian particles with mean field interaction. Z. Wahrsch. Verw. Gebiete 69 (1985) 439-459.

[18] B. Simon, Trace ideals and their applications. London Mathematical Society Lecture Notes series 35, Cambridge University press (1977)

[19] A.-S. Sznitman, Non linear reflecting diffusion process and the propagation of chaos and fluctuations associated.

[20] H. Tanaka, Limit Theorems for certain diffusion processes. in Proc. of the Taniguchi Symp, Katata (1982) 469-488, Tokyo, Kinokuniya (1984). J. Funct. Anal. 56 (1984) 311-336. 